\title{
Podocyte-associated talin1 is critical for glomerular filtration barrier maintenance
}

\author{
Xuefei Tian, ${ }^{1}$ Jin Ju Kim, ${ }^{2}$ Susan M. Monkley, ${ }^{3}$ Nanami Gotoh, ${ }^{1}$ Ramiro Nandez, 4,5,6 Keita Soda, ${ }^{1}$ \\ Kazunori Inoue, ${ }^{1}$ Daniel M. Balkin, ${ }^{4,5,6}$ Hossam Hassan, ${ }^{1}$ Sung Hyun Son, ${ }^{1}$ Yashang Lee, ${ }^{1}$ \\ Gilbert Moeckel,7 David A. Calderwood, ${ }^{4,8}$ Lawrence B. Holzman, ${ }^{9}$ David R. Critchley, ${ }^{3}$ \\ Roy Zent, ${ }^{10}$ Jochen Reiser, ${ }^{11}$ and Shuta Ishibe ${ }^{1}$
}

\begin{abstract}
${ }^{1}$ Department of Internal Medicine, Yale University School of Medicine, New Haven, Connecticut, USA. 2Department of Internal Medicine, University of Miami, Miami, Florida, USA. ${ }^{3}$ Department of Biochemistry, University of Leicester, Leicester, United Kingdom. ${ }^{4}$ Department of Cell Biology,

${ }^{5}$ Howard Hughes Medical Institute, ${ }^{6}$ Program in Cellular Neuroscience, Neurodegeneration, and Repair, ${ }^{7}$ Department of Pathology, and

${ }^{8}$ Department of Pharmacology, Yale University School of Medicine, New Haven, Connecticut, USA. 'Department of Internal Medicine, University of Pennsylvania, Philadelphia, Pennsylvania, USA. ${ }^{10}$ Department of Medicine, Vanderbilt University and Veterans Affairs Hospital, Nashville, Tennessee, USA. ${ }^{11}$ Department of Internal Medicine, Rush University Medical Center, Chicago, Illinois, USA.
\end{abstract}

\begin{abstract}
Podocytes are specialized actin-rich epithelial cells that line the kidney glomerular filtration barrier. The interface between the podocyte and the glomerular basement membrane requires integrins, and defects in either $\alpha_{3}$ or $\beta_{1}$ integrin, or the $\alpha_{3} \beta_{1}$ ligand laminin result in nephrotic syndrome in murine models. The large cytoskeletal protein talin 1 is not only pivotal for integrin activation, but also directly links integrins to the actin cytoskeleton. Here, we found that mice lacking talin1 specifically in podocytes display severe proteinuria, foot process effacement, and kidney failure. Loss of talin 1 in podocytes caused only a modest reduction in $\beta_{1}$ integrin activation, podocyte cell adhesion, and cell spreading; however, the actin cytoskeleton of podocytes was profoundly altered by the loss of talin1. Evaluation of murine models of glomerular injury and patients with nephrotic syndrome revealed that calpain-induced talin1 cleavage in podocytes might promote pathogenesis of nephrotic syndrome. Furthermore, pharmacologic inhibition of calpain activity following glomerular injury substantially reduced talin 1 cleavage, albuminuria, and foot process effacement. Collectively, these findings indicate that podocyte talin 1 is critical for maintaining the integrity of the glomerular filtration barrier and provide insight into the pathogenesis of nephrotic syndrome.
\end{abstract}

\section{Introduction}

The glomerular filtration barrier is composed of podocytes and fenestrated endothelial cells separated by a glomerular basement membrane (GBM), which together function to filter plasma to generate urine devoid of large molecular weight proteins. A breach of this barrier at any level can result in nephrotic syndrome, a severe kidney disease characterized by massive protein loss in the urine. Podocyte-GBM interactions are mediated by heterodimeric transmembrane integrin adhesion receptors that connect the extracellular matrix with the actin cytoskeleton. There are 24 distinct integrins, consisting of 1 of $18 \alpha$ subunits and 1 of $8 \beta$ subunits. Integrins are classified depending on their ligand preferences and the principal integrin that mediates podocytes adhesion to the GBM is the laminin-binding integrin $\alpha_{3} \beta_{1}(1)$. The importance of integrins in podocyte biology is underscored by the observation that in mice, podocyte-specific deletion of $\beta_{1}$ or $\alpha_{3}$ integrin develops into fatal proteinuria (2-4). Furthermore, deletion of the tetraspanin CD151 that interacts with integrin $\alpha_{3} \beta_{1}$ and the $\alpha_{3} \beta_{1}$ ligand $\beta_{2}$ laminin results in nephrotic syndrome $(4,5)$. Moreover, activation of $\alpha_{V} \beta_{3}$ integrin by podocytes or circulating ligands can cause specific glomerular diseases such as focal and segmental glomerulosclerosis (6). Integrin heterodimer clustering and the assembly of multiprotein cytoplasmic adhesion complexes termed focal adhesions are necessary for integrins to

Authorship note: Xuefei Tian and Jin Ju Kim contributed equally to this work. Conflict of interest: Jochen Reiser is an inventor on pending and issued patents on technologies concerning proteinuric kidney diseases.

Citation for this article: J Clin Invest. 2014;124(3):1098-1113. doi:10.1172/JCI69778 generate high-affinity adhesion or to relay intracellular signals (7). There are numerous proteins found within the focal adhesions, including proteins such as integrin-linked kinase (ILK), which has been demonstrated to play a critical role in podocyte development and function (8-10). Another critical protein required for integrin function found in focal adhesions is talin, a large $270 \mathrm{kDa}(2541$ a) dimeric adaptor protein that has been demonstrated to be critical in linking integrins to the actin cytoskeleton (11). Talin is comprises an atypical $\mathrm{N}$-terminal FERM domain (the talin head; THD) with 4 subdomains, F0, F1, F2, and F3, linked to a large C-terminal flexible rod $(12,13)$. The F3 subdomain adopts a phosphotyrosinebinding (PTB) domain fold that interacts with $\beta$ integrin tails and is required to activate integrins (14). THD has also been demonstrated to interact with the type 1 isoform of phosphatidylinositol phosphate kinase $\gamma($ PIPK $\gamma)(15-17)$ and phosphoinositides (18). Conversely, the talin rod comprises a series of amphipathic helical bundles, a number of which contain binding sites for integrins (19), actin (20), and cytoskeletal proteins such as vinculin (21). Cells lacking talin 1 have reduced cell adhesion, spreading, and migration, indicating its fundamental importance in focal adhesion assembly (22). Moreover, talin 1 can also undergo calpainmediated proteolytic cleavage-liberating THD and rod-promoting focal adhesion turnover (23).

While previous work has established a role for both integrins and actin-regulating proteins in podocyte biology (24-30), the key link between these proteins remains undetermined. Consequently, the goal of this study was to address the role of talin1, a key integrinand actin-binding protein, in the development and stability of the glomerular filtration barrier. To dissect its functional role, we gen- 
A
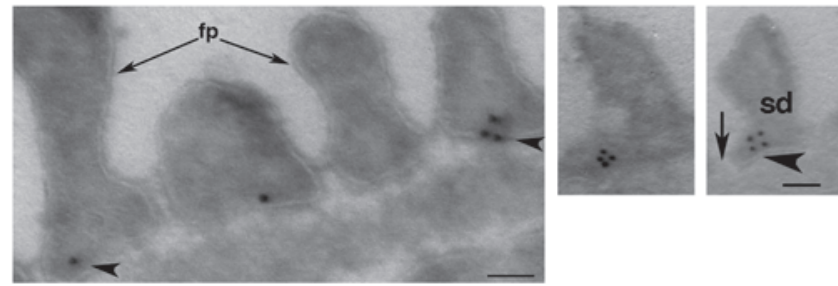

B

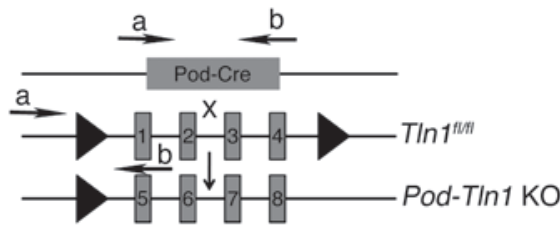

C

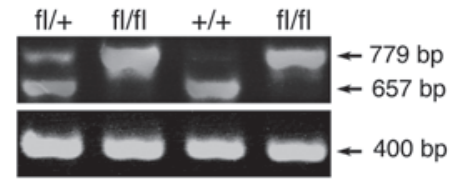

D

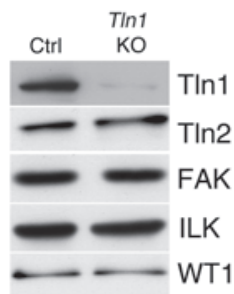

E
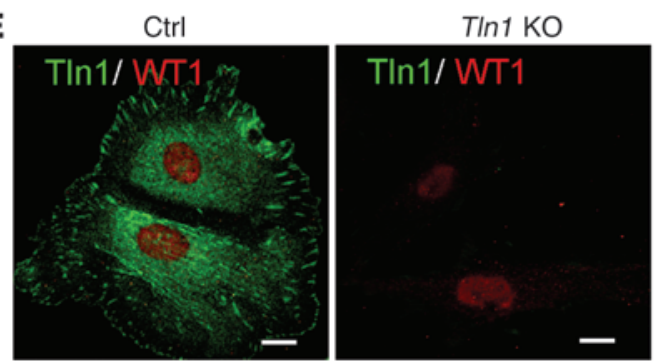

F
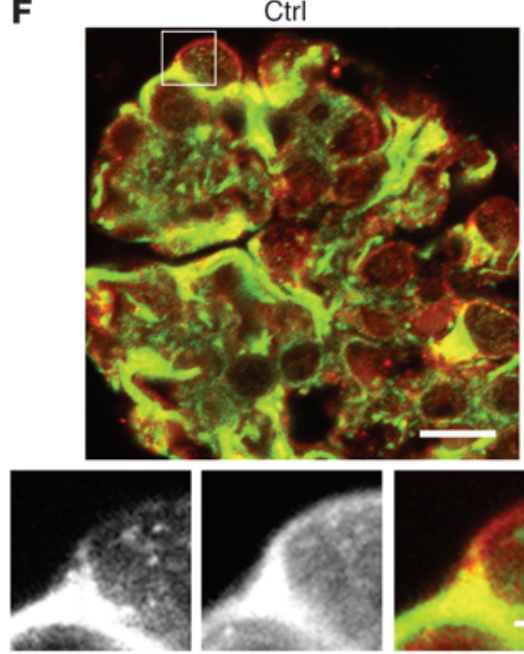

TIn1

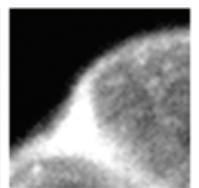

Nephrin

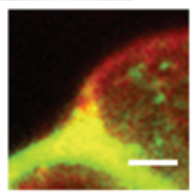

Merge

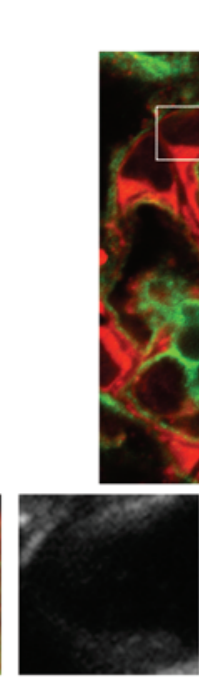

TIn1
TIn1 KO

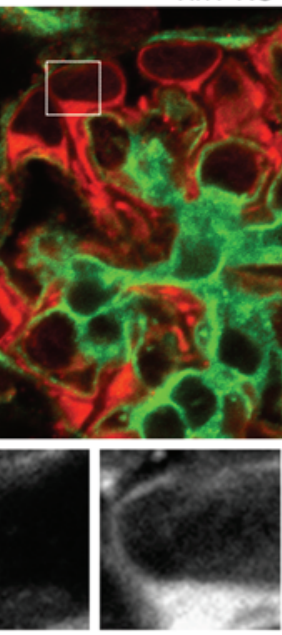

Nephrin

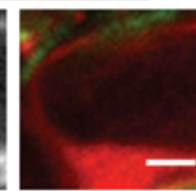

Merge

\section{Figure 1}

Generation of podocyte-specific TIn1-KO mice. (A) Immunogold transmission EM staining demonstrates talin1 (arrowheads) localizes to the base of the foot process (fp) and adjacent to the slit diaphragm (SD) (arrows) of podocytes from WT mouse kidneys. Scale bars: $100 \mathrm{~nm}$. (B) Schematic demonstrating breeding of the Podocin-Cre mice with $T \ln 1^{f l / f l}$ mice to generate podocyte-specific TIn1-KO mice (Pod-TIn1-KO). Forward and reverse primers are denoted as a and b, respectively, for Cre and $\operatorname{TIn} 1$. (C) Identification of TIn1 and Podocin-Cre by tail genotyping (age P7). (D and E) Talin1 expression in purified control (Ctrl) podocytes and lack of talin1 expression in podocytes harvested from Pod-TIn1-KO mice (age P7), as detected by Western blotting. (D) and immunofluorescence (E). Note that Ctrl podocytes express both talin1 and talin2. Podocytes were plated on collagen type I-coated glass coverslips and stained for WT1 (red) and for talin1 using an isoformspecific talin1 monoclonal Ab. Scale bars: 10 $\mu \mathrm{m}$. (F) Double-immunofluorescence detection of nephrin (red) and talin1 (green) on kidney sections of the indicated genotypes (age P14). Scale bars: $10 \mu \mathrm{m}$ (upper panels) $2 \mu \mathrm{m}$ (lower panels). erated a conditional podocyte-specific $T \ln 1-\mathrm{KO}$ mouse. We showed that loss of talin 1 specifically in podocytes resulted in a dramatic disruption of the glomerular permeability barrier and depleting talin 1 in podocytes resulted in a striking disorganization of the actin network. We further demonstrated that podocyte injury in mouse models of glomerular disease revealed an unexpected cleavage of talin 1 in the glomerulus due to an increase in the activity of calpain, a calcium-activated cysteine protease. In addition, we determined that in human samples from patients with focal segmental glomerulosclerosis (FSGS) or minimal change disease (MCD), there was a marked increase in calpain activity in the urine, akin to what was observed in our mouse models of glomerular injury. Finally, using a rabbit-anti-mouse GBM (NTS) model of glomerular injury, we showed that treatment with calpain inhibitors resulted in a reduction in proteinuria and foot process effacement. Collectively, our results not only implicate talin 1 as a critical component for the development and maintenance of podocytes by linking integrins to actin, but also suggest that calpain-mediated talin 1 cleavage may play a vital role in the pathogenesis of nephrotic syndrome following insult to the glomerular filtration barrier.

\section{Results}

Generation of podocyte-specific $T \ln 1-K O$ mice. As a first step toward investigating the role of talin 1 in the glomerulus, we used immunogold electron microscopy (EM) to determine its localization. Talin 1 localized primarily at the basal aspect of the podocytes (Figure 1A). Furthermore, in a subset of podocytes, talin1 was also found clustered at the base of the podocytes adjacent to 
A
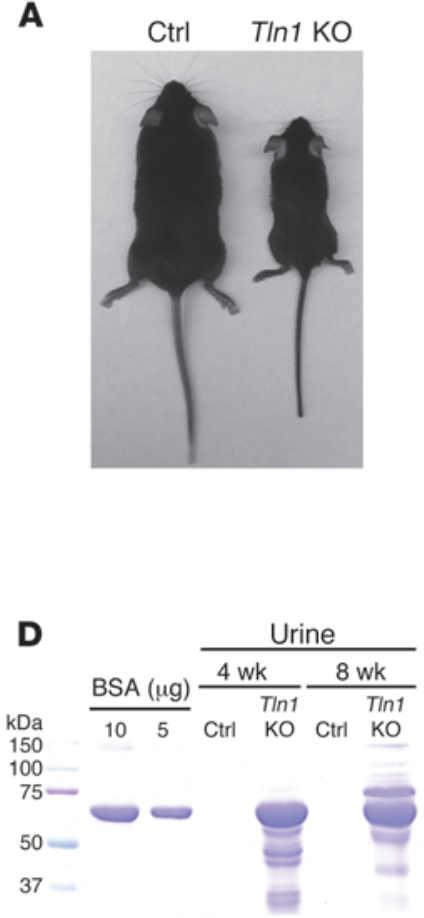

$\mathbf{F}$

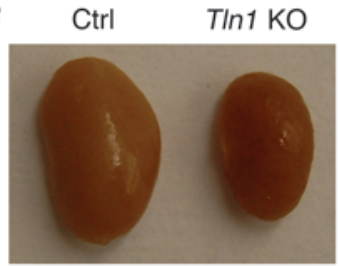

B

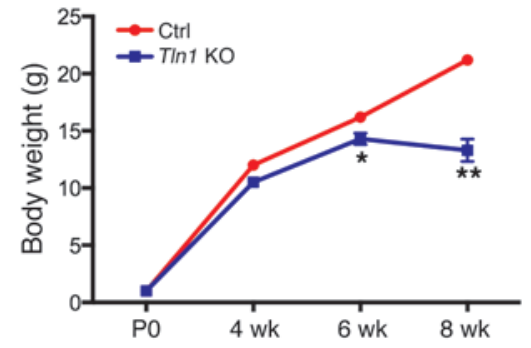

C

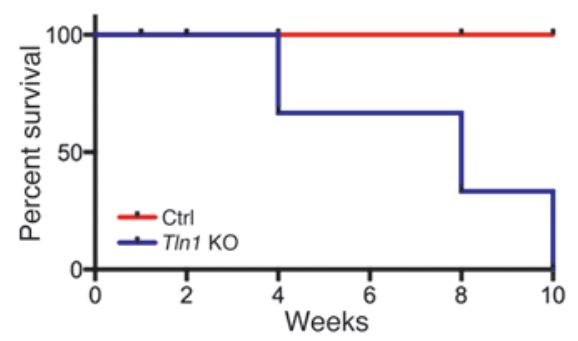

E.
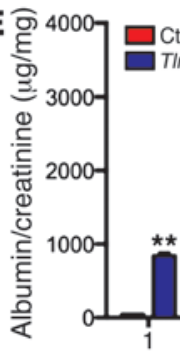

\section{trl}

Tini KO
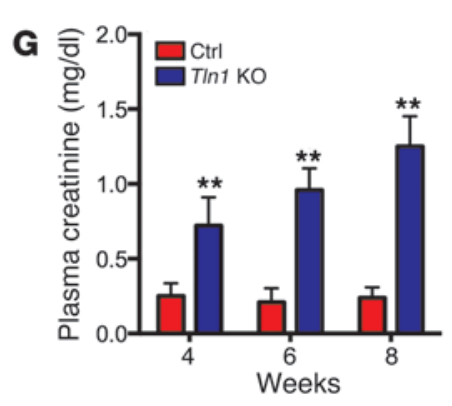

\section{Figure 2}

Loss of talin1 in podocytes results in severe proteinuria and kidney failure. (A) Representative images of control and Pod-TIn1-KO mice at 8 weeks of age. (B) Pod-TIn1-KO mice, relative to controls, fail to gain weight by 4 weeks of age. $n=10$ mice. ${ }^{*} P<0.01$; ${ }^{* *} P<0.001$. (C) Survival curve of Pod-TIn1-KO mice demonstrates greater than $70 \%$ death by 8 weeks of age. $n=10$ mice. (D) SDS-PAGE (Coomassie blue staining) of standard BSA (numbers indicate $\mu \mathrm{g}$ ) and of urine from Pod-TIn1-KO mice, demonstrating albuminuria at 4 and 8 weeks of age. Equal volumes of standard BSA and urine $(2 \mu \mathrm{l})$ were loaded in each lane. (E) Quantification of urinary albumin normalized to creatinine at $1,2,4,6$, and 8 weeks of age. $n=8$ mice. (F) Kidneys of Pod-TIn1-KO mice are paler and smaller than those of controls and have a corrugated appearance at 8 weeks of age. (G) Elevated plasma creatinine in Pod-TIn1-KO mice at 4, 6, and 8 weeks of age. $n=5$ mice. ${ }^{* \star} P<0.001$.

Loss of podocyte talin1 results in proteinuria and kidney failure. Pod-Tln1-KO mice appeared normal at birth, but by 4 weeks of age, their weight gain fell below that of $T \ln 1^{f l / f l}$ littermate controls. By 8 weeks of age, the animals were smaller, hunched, and severely lethargic (Figure 2, A and B), with death resulting in more than $70 \%$ of mice by 8 weeks of age (Figure 2C). As vertebrates contain 2 highly conserved talin isoforms (talin 1 and talin2), we also examined Pod-Th1-KO mice crossed with constitutive Th2-KO mice (33), Intriguingly, ablation of podocyte talin 1 in mice lacking talin 2 (33), which is also expressed in podocytes (Figure 1D and Supplemental Figure 1, A and B), did not significantly change the Pod-Tln1-KO phenotype (data not shown), suggesting that talin 1 function most likely predominates in normal podocyte physiology. Urine analysis on Pod-Tln1-KO mice using SDS-PAGE followed by Coomassie blue staining demonstrated robust albuminuria (Figure 2D) beginning as early as postnatal week 1 and

the slit diaphragm (Figure 1A). Global loss of the $T \ln 1$ gene is embryonic lethal (31), so to study the role of talin 1 in podocyte biology, we generated a podocyte-specific $T \ln 1$-KO using the CreLox system based on podocin-Cre, which drives Cre expression at E13-E14 (32). Compound $\operatorname{Tn} 1$ heterozygote mice were mated to generate podocyte-specific $T \ln 1-\mathrm{KO}$ mice (Pod-Tln1-KO) (Figure 1B). Pod-Tln $1-\mathrm{KO}, \operatorname{Pod}-T \ln 1^{f l /+}$, and $T \ln 1^{f l / f l}$ mice (used as controls) were born according to the expected Mendelian frequency as identified by tail genotyping (Figure 1C). Western blot analysis and immunofluorescence revealed markedly diminished expression of talin 1 in the Dynabeads-extracted podocyte-enriched cell fractions obtained from the Pod-Tln1-KO mice (Figure 1, D and E), but no difference was observed in expression of talin 2 in the podocytes (Figure 1D and Supplemental Figure 1, A and B; supplemental material available online with this article; doi:10.1172/ JCI69778DS1). Moreover, loss of podocyte talin1 immunoreactivity was also demonstrated in the Pod-Tln1-KO kidney sections costained with the podocyte-specific marker nephrin (Figure 1F). continuing to progress, as validated by ELISA, with normalization to urine creatinine (Figure 2E). Furthermore, plasma creatinine measurements in Pod-Tln1-KO mice demonstrated severe kidney failure when compared with littermate controls (Figure 2G), while the harvested kidney took on a shrunken and corrugated appearance at 8 weeks of age (Figure $2 \mathrm{~F}$ ).

Loss of podocyte talin 1 results in proteinuria and foot process effacement. Histological examination of Pod-Tln1-KO kidneys revealed normal features at birth. However, by 2 weeks of age, approximately $15 \%$ of glomeruli revealed severe glomerular capillary dilatation (Figure 3A; quantified in Figure 3B). By 8 weeks of age, the glomeruli had undergone a process of global sclerosis (Figure 3A; quantified in Figure 3C) and the kidney further demonstrated severe interstitial fibrosis, tubular dilatation, and proteinaceous casts (Figure 3D; characteristics of the glomerulus, both scanning and transmission EM were performed on Pod-Tln1-KO and control kidneys. In Pod-Th1-KO mice 10 days following birth, scanning EM analysis quantified in Figure 3E). To further determine the ultrastructural 
A
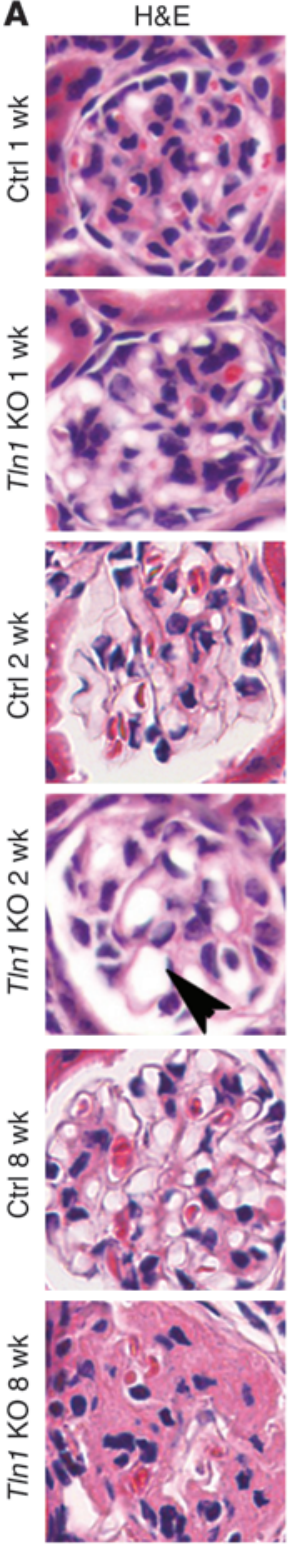

B

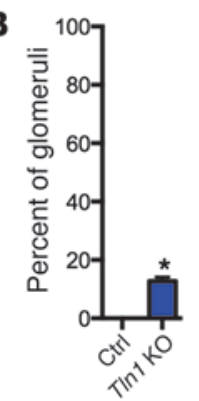

PAS
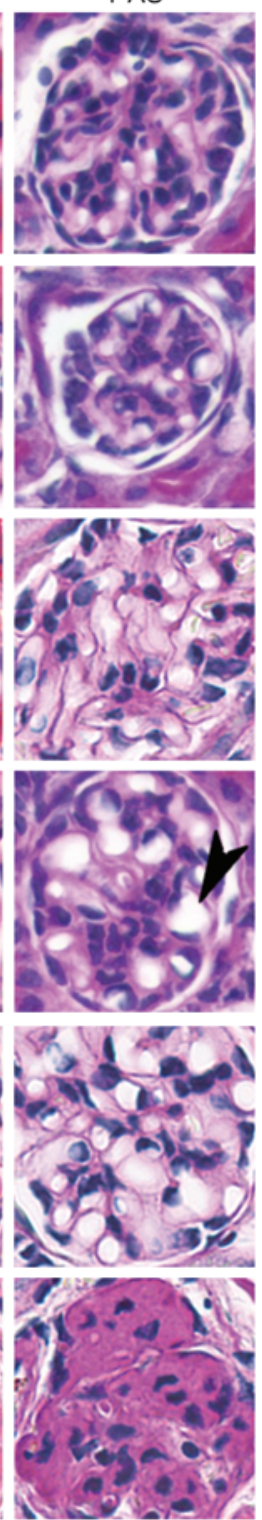

C

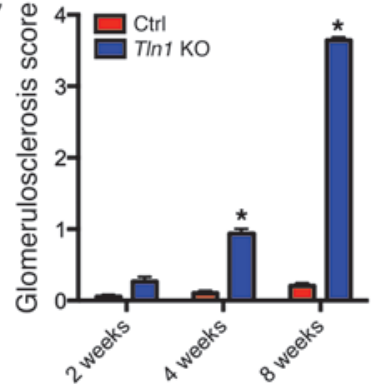

Trichrome
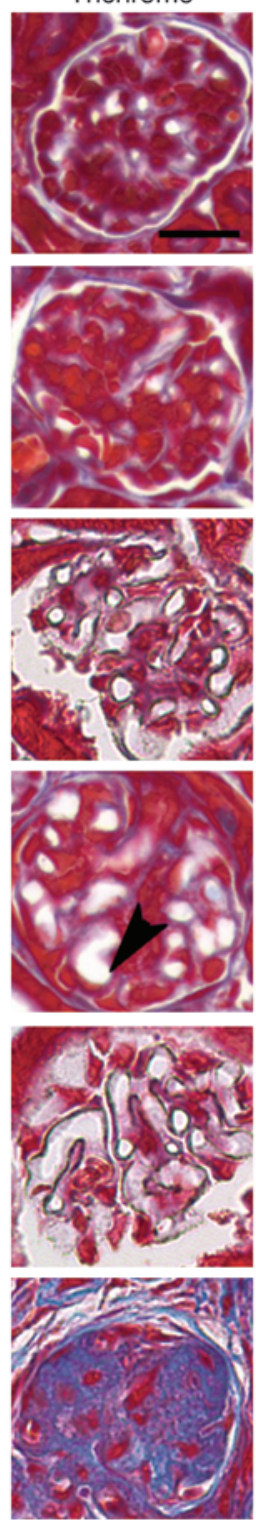
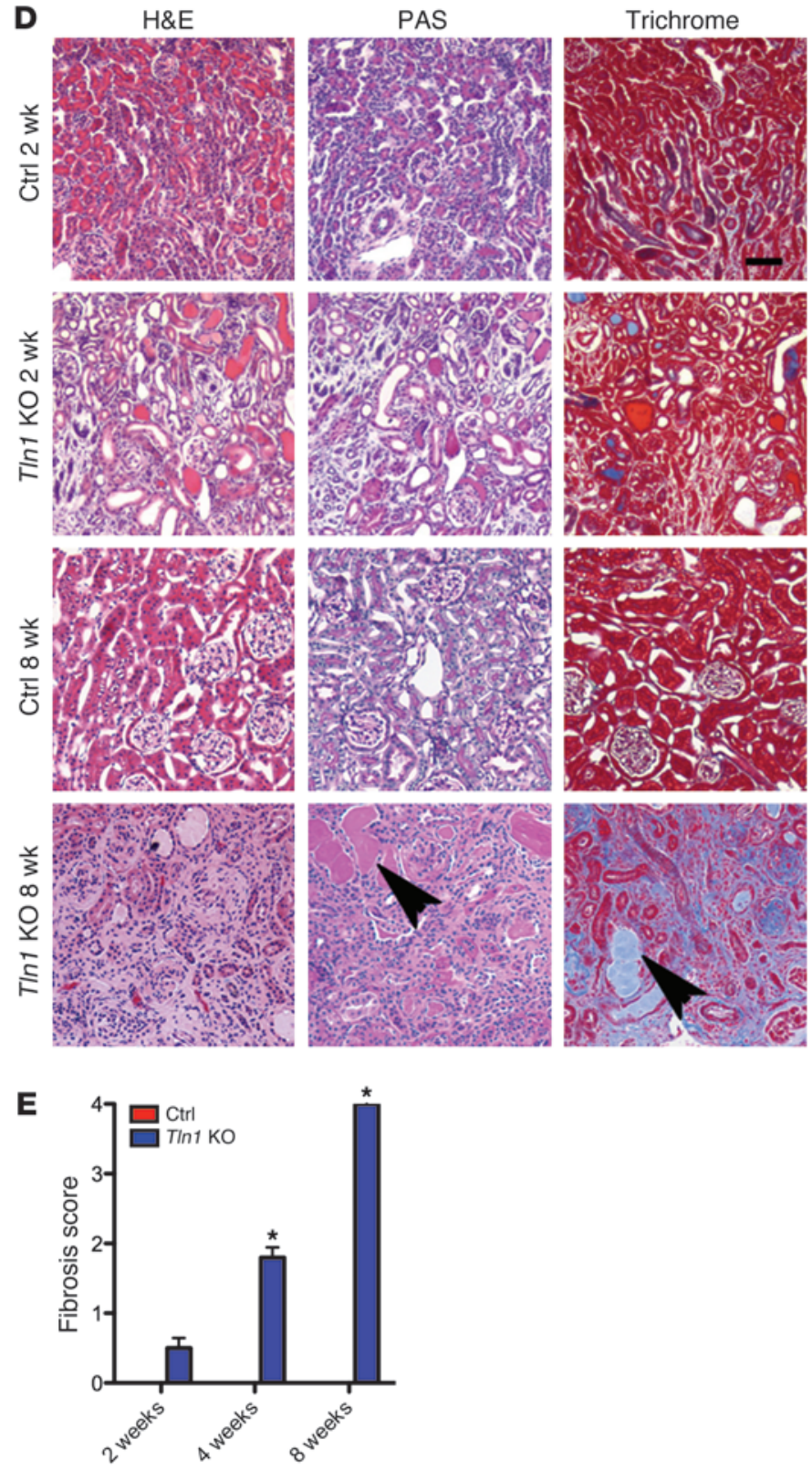

Figure 3

Podocyte-specific loss of talin1 results in glomerular capillary loop dilation with progressive glomerulosclerosis (scarring and fibrosis of the glomerulus) and interstitial fibrosis. (A) Representative light microscopy images (H\&E, PAS, and trichrome) of glomeruli from Pod-TIn1-KO mice revealing histological evidence of dilated glomerular capillary loops at 2 weeks of age (arrowheads), which progresses to diffuse glomerulosclerosis by 8 weeks. Scale bar: $25 \mu \mathrm{m}$. (B) Quantification as percentage of glomeruli, with capillary loop dilation at 2 weeks of age, in Pod-Tln1-KO mouse kidney sections when compared with control. $n=3$ mice. (C) Quantification of glomerulosclerosis at 2, 4, and 8 weeks of age. $n=4$ mice (D) Proteinaceous casts (arrowhead), dilated tubules, and severe interstitial fibrosis are observed in Pod-TIn1-KO mice at 8 weeks of age. Scale bar: $50 \mu \mathrm{m}$. (E) Quantification of interstitial fibrosis at 2, 4, and 8 weeks of age. $n=4$ mice. ${ }^{*} P<0.001$. 
A
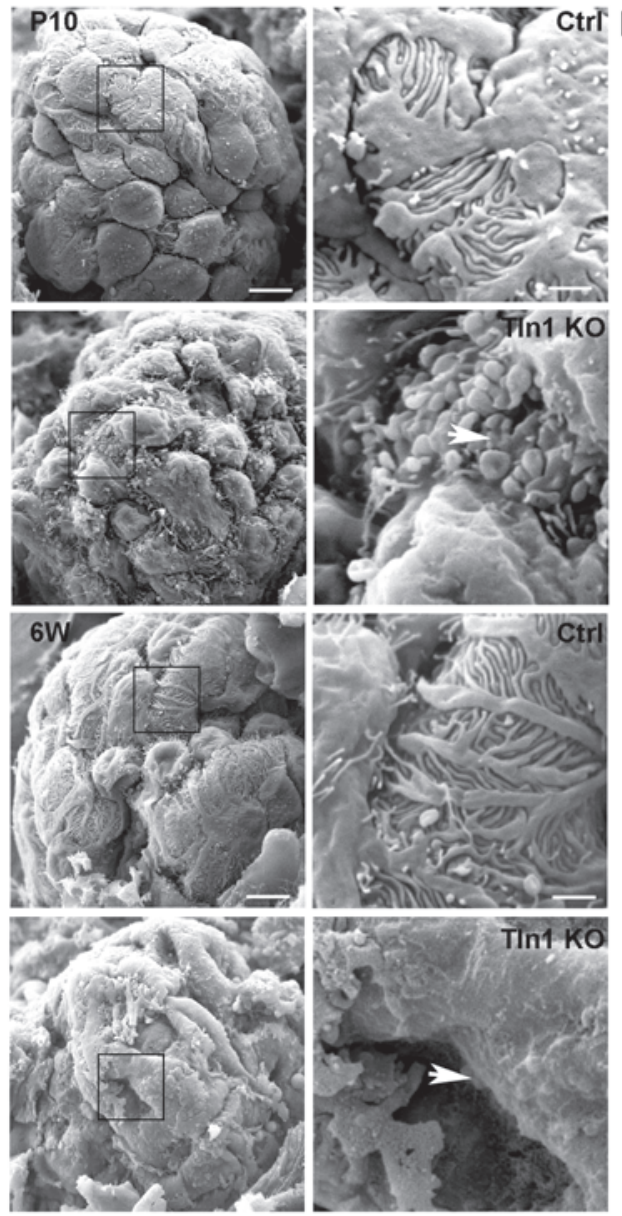

C

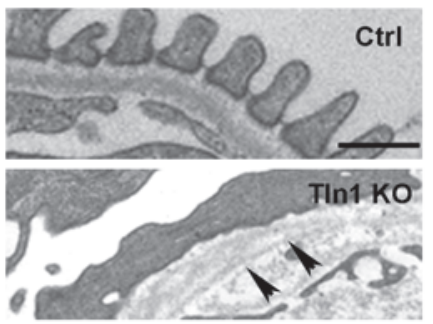

D

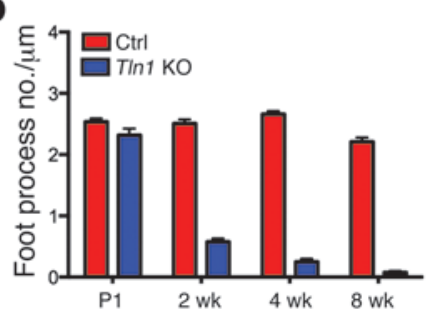

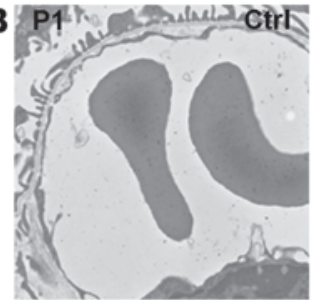
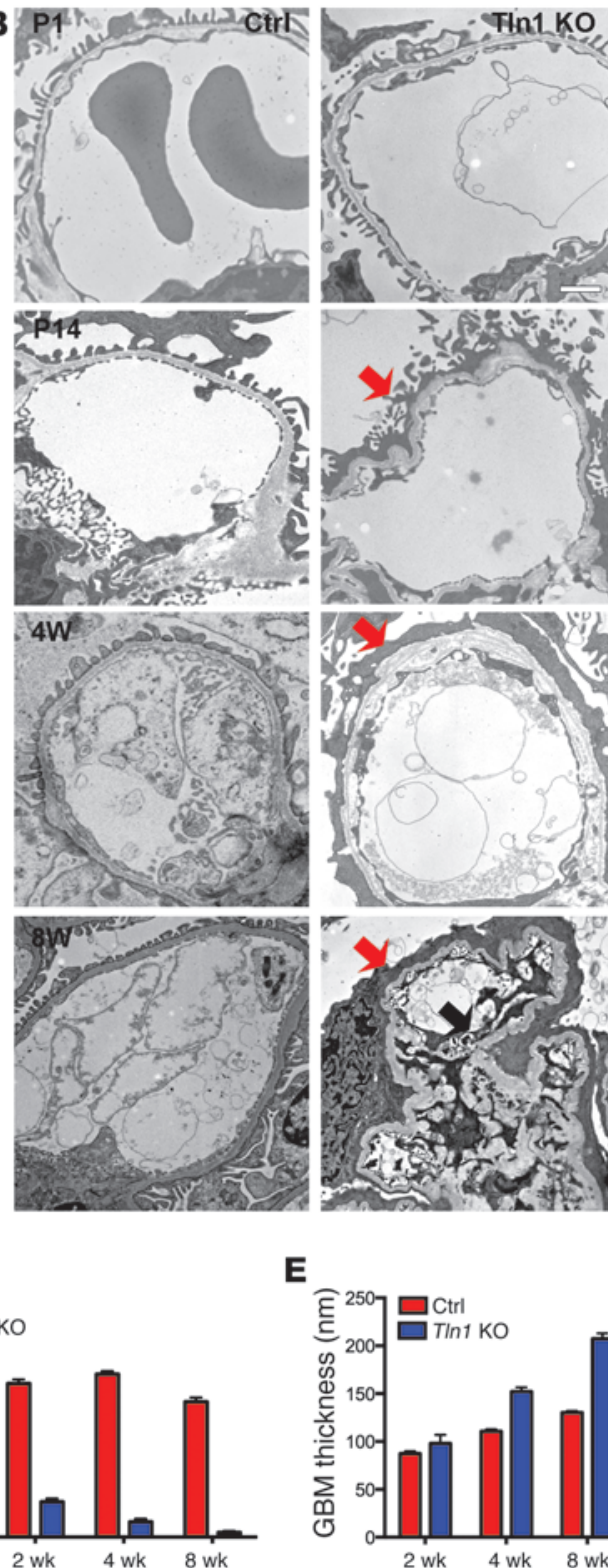

E

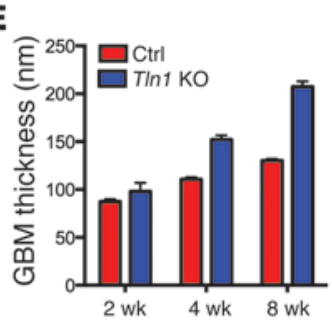

\section{Figure 4}

Podocyte-specific loss of talin1 results in foot process effacement. (A) Scanning electron micrographs illustrating the ultrastructure of glomerulus of control and Pod-TIn1-KO mice at P10 and at 6 weeks. Arrowheads demonstrate loss of foot process interdigitations. Scale bars: $5 \mu \mathrm{m}$ (left panels); $1 \mu \mathrm{m}$ (right panels). (B) Transmission electron micrographs illustrating foot process effacement by 2 weeks of age (P1, P14, 4 weeks, and 8 weeks). Scale bar: $1 \mu \mathrm{m}$. The red arrows depict foot process effacement while the black arrow depicts mesangial expansion. (C) High-magnification micrographs from control and mutant glomeruli showing thickening and splitting of the basement membrane (4 weeks after birth), as indicated by the arrowheads. Scale bar: $200 \mathrm{~nm}$. Note the effacement (podocyte collapsing on its basement membrane in the Pod-TIn1-KO mice). (D) Quantification of the number of foot processes per micrometer of GBM in the control and PodTIn1-KO mice at various ages. $n=2$ mice at each time point. (E) Quantification of GBM thickness in the control and Pod-TIn1-KO mice at various ages. $n=2$ mice at each time point. demonstrated a dramatic loss of podocyte foot process interdigitations and destruction of the major processes (Figure 4A). Interestingly, the transmission EM of glomeruli appeared normal at birth (P1) (Figure 4B), but by postnatal week 2, there was significant foot process effacement (Figure 4B; quantified in Figure 4D), validating our findings observed from the scanning EM. By 4 weeks of age, the architecture of the podocyte-endothelial interface was strikingly different from the classical architecture observed in age-matched controls (Figure 4B). In the Pod-Tln1-KO mice, there was a profound basement membrane splitting (Figure 4C), and by 8 weeks of age, thickening of the basement membrane and mesangial expansion were observed (Figure 4B; quantified in Figure 4E).

Loss of podocyte talin 1 results in a modest reduction in $\beta_{1}$ integrin activation and cell spreading. Given that the Pod-Th1-KO mice demonstrated severe proteinuria as early as 1 week of age, we decided to examine the possibility that podocyte loss might occur in the Pod-Tln1-KO mice as a result of cell detachment and therefore generate defects in the glomerular filtration barrier. Compared with those from littermate control $\left(\operatorname{Tn} 1^{f l f l}\right)$ mice, kidney sections from P14 Pod-Tln1-KO mice, stained with the podocyte-specific transcription factor WT1, revealed similar podocyte numbers (Figure 5A; quantified in Figure 5B). Further validating our results, TUNEL staining demonstrated no evidence of apoptosis occurring within the Pod-Tln1-KO mice glomeruli, although increased apoptosis within the tubular segments was observed (Figure 5C). Talin 1 binding to the cytoplasmic tail of the $\beta$ integrin subunit is important for integrin activation $(34,35)$. Furthermore, as the podocyte-specific loss of $\beta_{1}$ integrin results in proteinuria, next we explored $\beta_{1}$ integrin expression and activation. No significant differences were observed in the expression levels of either $\beta_{1}$ or $\alpha_{3}$ integrin from enriched primary podocytes harvested from $\operatorname{Tn} 1^{f l / f l}$ and Pod-Tln1-KO mice (Figure 5D). We also detected no differences in the $\beta_{1}$ or $\alpha_{3}$ integrin localization within the glomerulus and podocytes following immunostaining (Supplemental Figure 2, A 
A

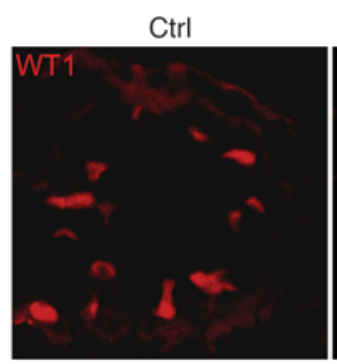

D

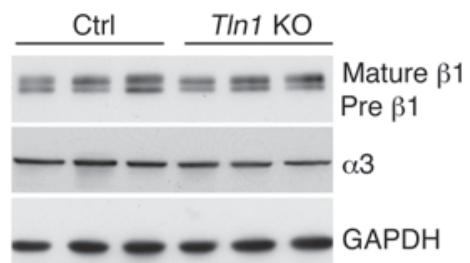

TIn1 KO

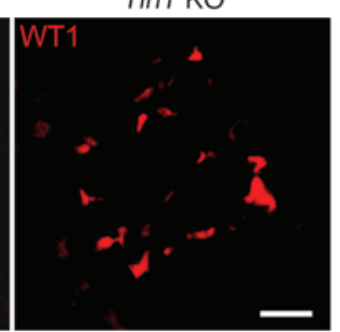

B
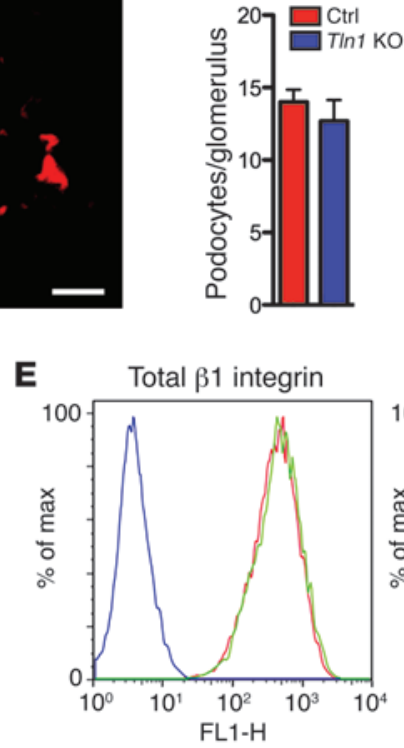

C

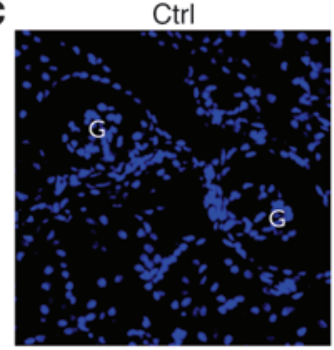

Tln1 KO

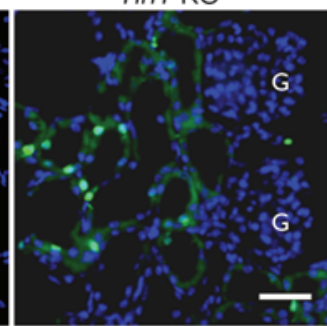

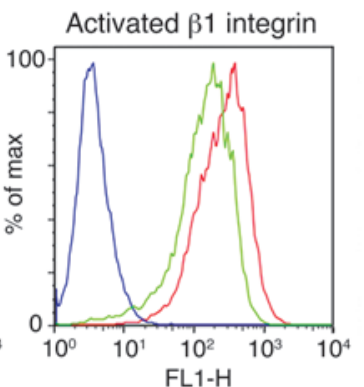

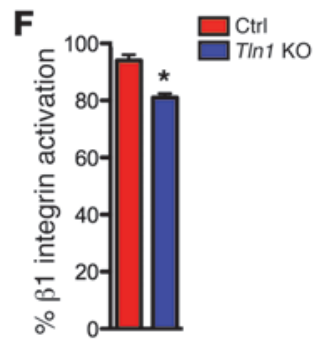

G

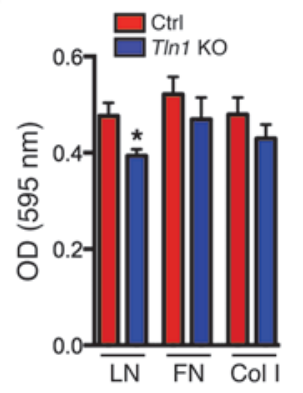

H

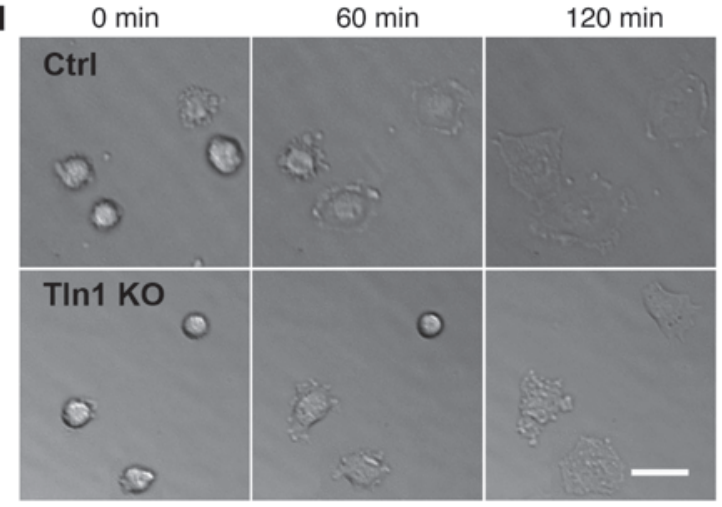

I

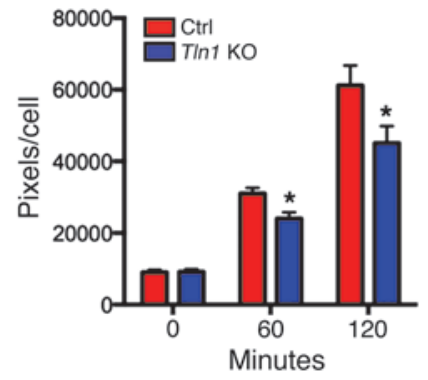

Figure 5

Lack of talin1 in podocytes results in reduced integrin activation, adhesion, and cell spreading. (A) Representative images of kidney sections from control and Pod-TIn1-KO mouse kidneys stained with WT1, a podocyte-specific transcription factor, at 2 weeks of age. Scale bar: $10 \mu \mathrm{m}$. (B) Quantification of WT1 staining per glomerulus in A. $n=6$ experiments. (C) TUNEL staining of kidney sections from control and Pod-TIn1-KO mice at 2 weeks of age. G, glomerulus. Scale bar: $20 \mu \mathrm{m}$. (D) Primary podocytes isolated from control and Pod-TIn1-KO kidneys immunoblotted for $\beta_{1}$ integrin, $\alpha_{3}$ integrin, and GAPDH. (E) Cell-surface expression of integrins on freshly isolated primary podocytes from control and PodTIn1-KO mice analyzed by FACS by staining with Abs against total $\beta_{1}$ integrin and 9EG7 epitope of active $\beta_{1}$ integrin. Pod-TIn1-KO podocytes are denoted in green, and control podocytes are denoted in red histograms. (F) Quantification of $\mathbf{E}$ expressed as percentage of $\beta_{1}$ integrin activation per total $\beta_{1}$ integrin. $n=4$ experiments. (G) Primary podocytes isolated from control and Pod-TIn1-KO mice demonstrate a small but significant decrease in adhesion to laminin (LN) and not to fibronectin (FN) or collagen type I (Col I). $n=4$ experiments. (H) Representative image of control and Pod-TIn1-KO podocytes plated on laminin and monitored by live cell imaging for 120 minutes. (I) Quantification of cell area was performed on 50 random cells in each experiment. $n=3$ experiments. Scare bar: $20 \mu \mathrm{m}$. ${ }^{*} P<0.01$.

and B). Moreover, podocytes from Pod-Tln1-KO mice showed only a moderate, albeit significant decrease in $\beta_{1}$ integrin activation as measured by binding of the activation-sensitive 9EG7 Ab (Figure 5E; quantified in Figure 5F). To analyze the effects of $T \ln 1-\mathrm{KO}$ on podocyte cell adhesion and spreading, primary podocytes isolated from control and mutant mice were seeded on plates coated with either fibronectin, laminin, or collagen type I. There was again a modest impairment in adhesion to laminin, but not to fibronectin or collagen type I (Figure 5G). Moreover, a modest reduction in cell spreading was observed (Figure 5H; quantified in Figure 5I) in the isolated mutant podocytes plated on laminin when compared with control but not to fibronectin or collagen type I (Supplemental Figure 2, C and D). However, there was no difference in the time course of integrin-dependent activation of pERK, pAKT, and p38MAPK at 10, 30, and 60 minutes on laminin as determined by Western blotting of podocytes isolated from Pod-Th1-KO and $T \ln 1^{f l / f l}$ mice (data not shown). The above results were further confirmed using WT podocytes exposed to lentivirus containing shRNAmir-Tln1 (Supplemental Figure 3A); Talin1 knockdown caused only a modest though significant reduction in cell spreading, supporting our results observed in the Pod-Tln1-KO podocytes (Supplemental Figure 3B; quantified in Figure 3C). 
A
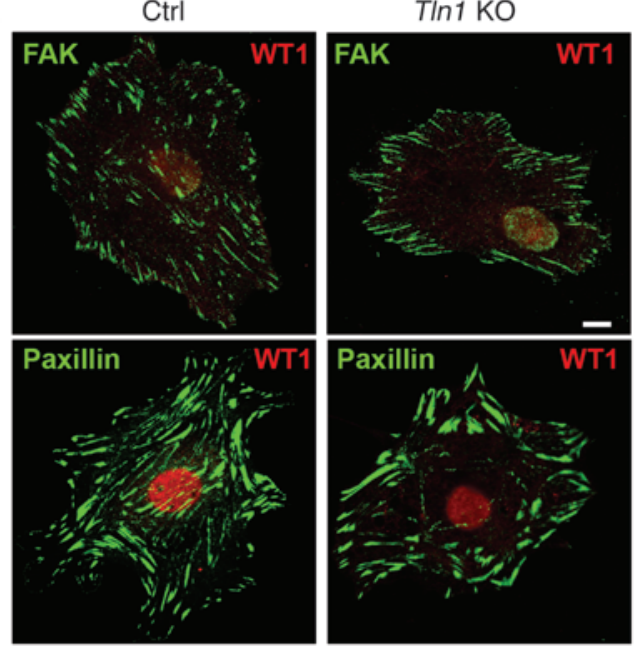

D

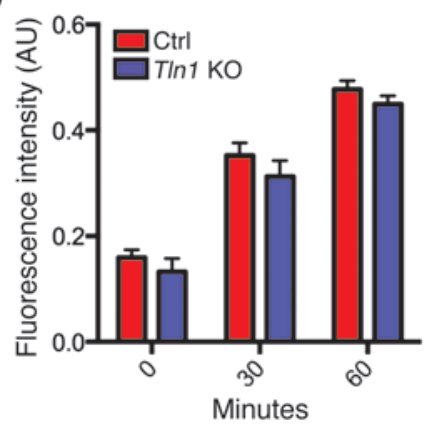

E
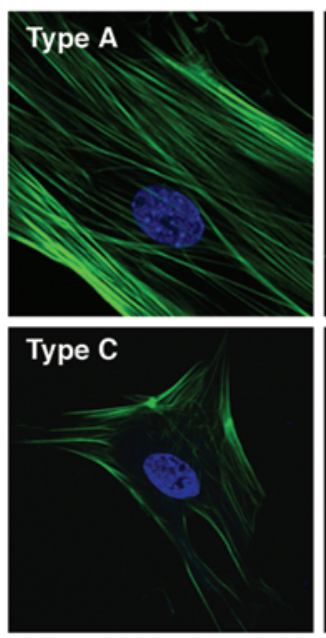

C
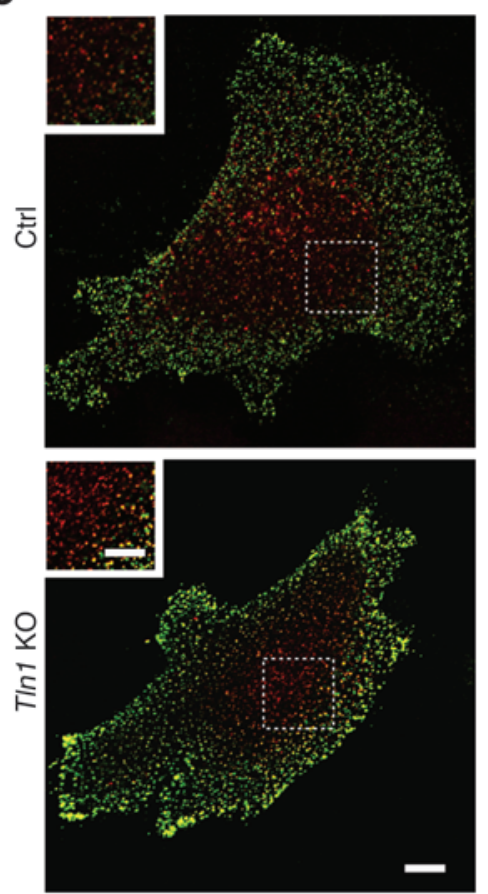

$\mathbf{F}$

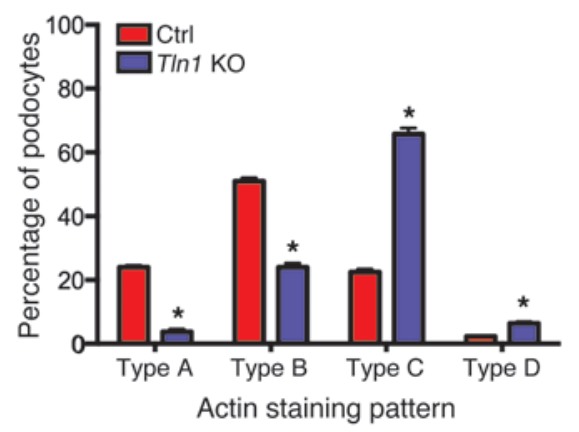

G

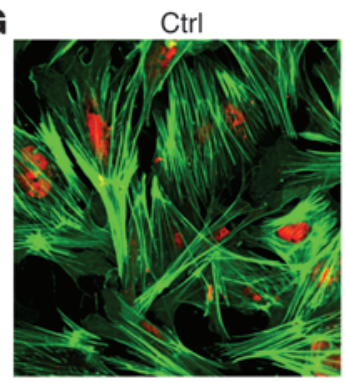

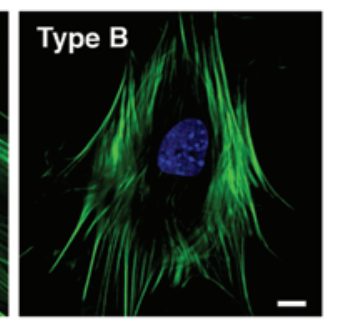
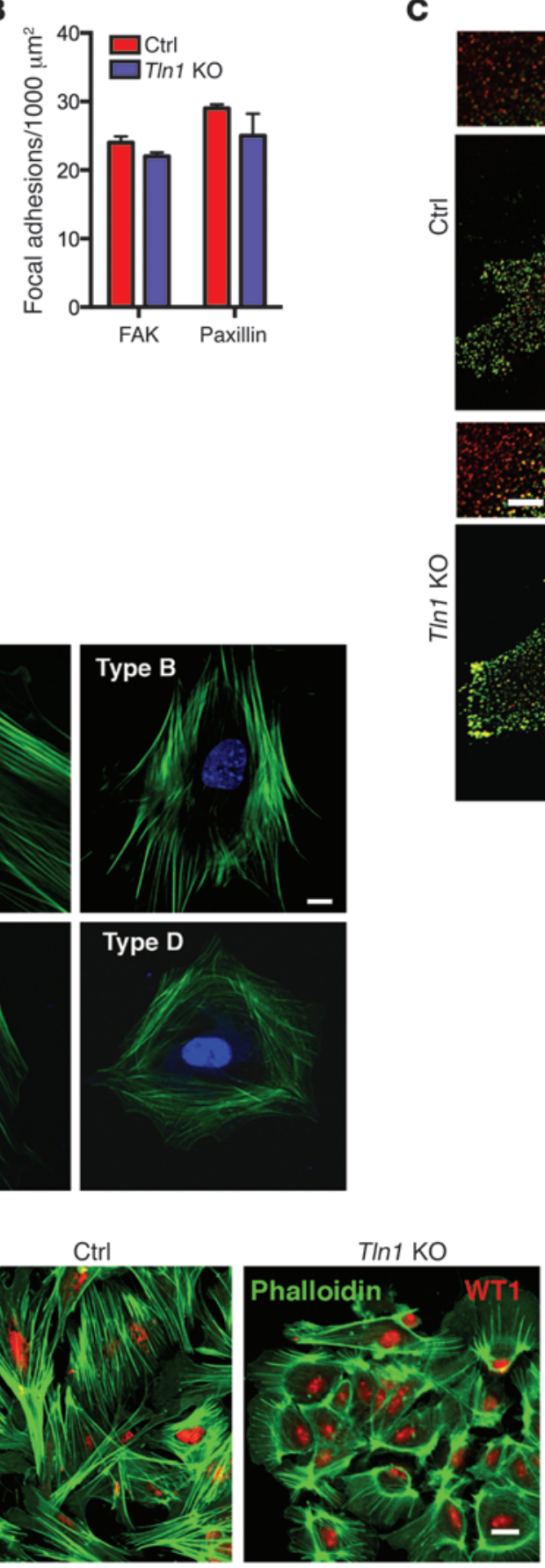

Figure 6

Actin stress fibers are reduced in Pod-TIn1-KO podocytes. (A) Representative images of paxillin and FAK in focal adhesions in isolated podocytes from control and Pod-TIn1-KO mice. Scale bar: $10 \mu \mathrm{m}$. (B) Quantification of focal adhesions represented as focal adhesions/1000 $\mu \mathrm{m}^{2}$ of podocyte cell area in control and Pod-TIn1-KO podocytes. 25 cells were analyzed in each experiment for control and Pod-TIn1-KO podocytes. $n=3$ experiments. (C) Representative image of internalized (red) versus cell surface CD8-nephrin chimera in control and TIn1-KO podocytes at 60 minutes. Scale bar: $10 \mu \mathrm{m} ; 3 \mu \mathrm{m}$ (inset). (D) Quantification of the data shown in C. ( $n=3$ experiments, 25 cells evaluated per experiment). (E) Representative images of different types of phalloidin staining patterns observed in isolated control podocytes. Scale bar: $10 \mu \mathrm{m}$. (F) Quantification of phalloidin staining in control and Pod-TIn1-KO podocytes scored by an observer blind to the genotype. The Pod-TIn1-KO podocytes had fewer cells with types A ( $>90 \%$ of cell area filled with thick cables) and B (at least 2 thick cables running under nucleus and rest of cell area filled with fine cables) staining patterns and more in the type $C$ (no thick cables, but some cables present) and type $D$ (no cables visible in the central area of the cell) pattern when compared with control podocytes. $n=3$ experiments. ${ }^{\star} P<0.001$. (G) Representative image of confluent control and Pod-TIn1-KO podocytes stained with phalloidin and WT1. Scale bar: $30 \mu \mathrm{m}$. 

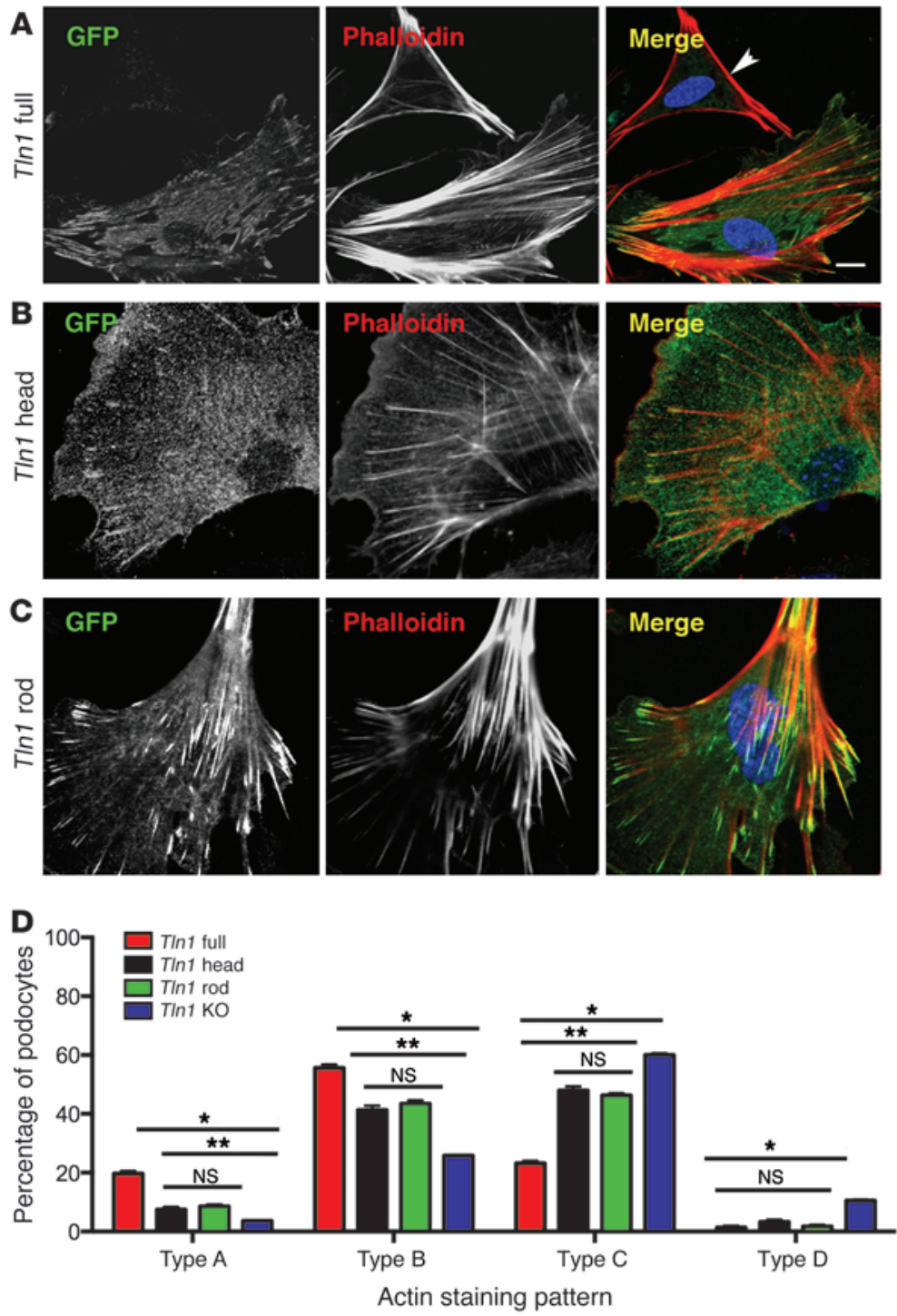

Loss of podocyte talin 1 results in defects of the actin cytoskeleton. To gain further insight into the function of talin 1 in podocytes, we initially evaluated the localization of focal adhesions as previously, the loss of talin 1 in cells has been shown to result in a mislocalization and a reduction of expression of focal adhesions (36). Thus we stained mouse primary podocytes for FAK and paxillin, 2 proteins localized at focal adhesions. However, the localization and expression of FAK and paxillin in the $T \ln 1^{f l f l}$ and $P o d-T \ln 1-\mathrm{KO}$ were very similar (Figure 6A; quantified in Figure 6B). Talin1 has also been demonstrated to interact with PIPK $\gamma$, the major phosphatidylinositol4,5-bisphosphate-synthesizing ([PI4,5P2]-synthesizing) enzyme (15). This interaction has been suggested to play an integral role in clathrin-mediated endocytosis (37), and we have shown through genetic mouse models of disease that perturbation of endocytic proteins results in severe proteinuria (38). To determine whether endocytic defects exist in the Pod-Tln1-KO podocytes, an internalization assay of nephrin was performed. Podocytes were transfected with a protein chimera comprising the transmembrane and cytoplasmic domain of nephrin and the extracellular domain of CD8. Using an anti-CD8 Ab-based internalization assay, we

\section{Figure 7}

Reexpression of full-length GFP-TIn1 in Pod-TIn1-KO podocytes results in stress fiber reconstitution. (A-C) Representative images of phalloidin staining (red) following reexpression of GFP-TIn1 full-length (residues 1-2541) (A), GFP-TIn1 head (residues 1-435) (B), and GFP-TIn1 rod (residues 435-2541) (C) in Pod-TIn1-KO podocytes. Note loss of stress fibers and prominent cortical actin staining in untransfected Pod-TIn1-KO podocytes (arrowhead). Scale bar: $10 \mu \mathrm{m}$. (D) Quantification of phalloidin staining in podocyte-specific TIn1-KO podocytes following reexpression of the different GFP-TIn1 constructs indicated in A-C. $n=4$ experiments ${ }^{*} P<0.01$, comparing GFP-TIn 1 full-length vs. GFP-TIn1 head, GFP-TIn1 rod, or Pod-TIn1-KO podocytes (untransfected); ${ }^{* *} P<0.01$, GFPTIn1 head or GFP-TIn1 rod vs. untransfected podocytes.

found that the internalization of this probe was not significantly delayed in the Pod-Tn1-KO podocytes when compared with control (Figure 6C; quantified in Figure 6D). Given that talin 1 has also been demonstrated to interact with and regulate the actin cytoskeleton (7), we explored the actin architecture in primary podocytes isolated from $T \ln 1^{f l / f l}$ and $\operatorname{Pod}-T \ln 1-\mathrm{KO}$ mice by staining with conjugated phalloidin-Alexa Fluor 488. Podocytes isolated from the Pod-Th1-KO mice revealed a striking reduction in actin stress fiber formation, with an accentuated cortical localization in both confluent and nonconfluent cells (Figure 6, E and G; quantified in Figure 6F). To investigate whether the talin $1 \mathrm{~N}$-terminal head or the $\mathrm{C}$-terminal rod plays an important role in the actin cytoskeleton in podocytes, GFP-Tln1 full-length (residues 1-2541), GFP$T \ln 1$ head (residues 1-435), or GFP-Tln1 rod (residues 435-2541) was reexpressed in Pod-Tln1-KO primary podocytes. As expected, the actin cytoskeleton defects in $\mathrm{Pod}-\mathrm{T} \ln 1-\mathrm{KO}$ primary podocytes were rescued by expressing GFP-Tln1 full-length talin1, although KO cells expressing either GFP-Tln 1 head or GFP-TIn 1 rod alone had partial effects, suggesting that both the $\mathrm{N}$ - and $\mathrm{C}$-terminal regions of talin 1 are required for actin stress fiber formation in podocytes (Figure 7, A-C; quantified in Figure 7D). We further utilized fluorescently labeled mCh-Utrophin, a marker for F-actin (39), to visualize in real time the actin dynamics of control and $T \ln 1-\mathrm{KO}$ primary podocytes. Results recapitulated the phalloidin-staining pattern with a severe reduction in stress fibers in $T \ln 1$-KO (Supplemental Videos 1 and 2).

Podocyte injury results in calpain-induced talin1 proteolysis. We next sought to determine the role of talin 1 following podocyte injury in an intact organism. Therefore, we perfused mice with protamine sulfate, an agent that has been shown to induce podocyte foot process effacement (40). Compared with $T \ln 1^{f l f l}$ mice glomeruli perfused with HBSS, glomeruli isolated from mice treated with protamine sulfate consistently demonstrated an unexpectedly striking talin1 cleavage product (Figure 8A; quantified in Supplemental Figure 4A). To further explore whether talin 1 cleavage within the kidney occurred following glomerular injury, we used another model where NTS was injected in mice, resulting in GBM immunoglobulin deposition (Figure 8B), proteinuria, and foot process effacement (data not shown). Mice treated with NTS also 

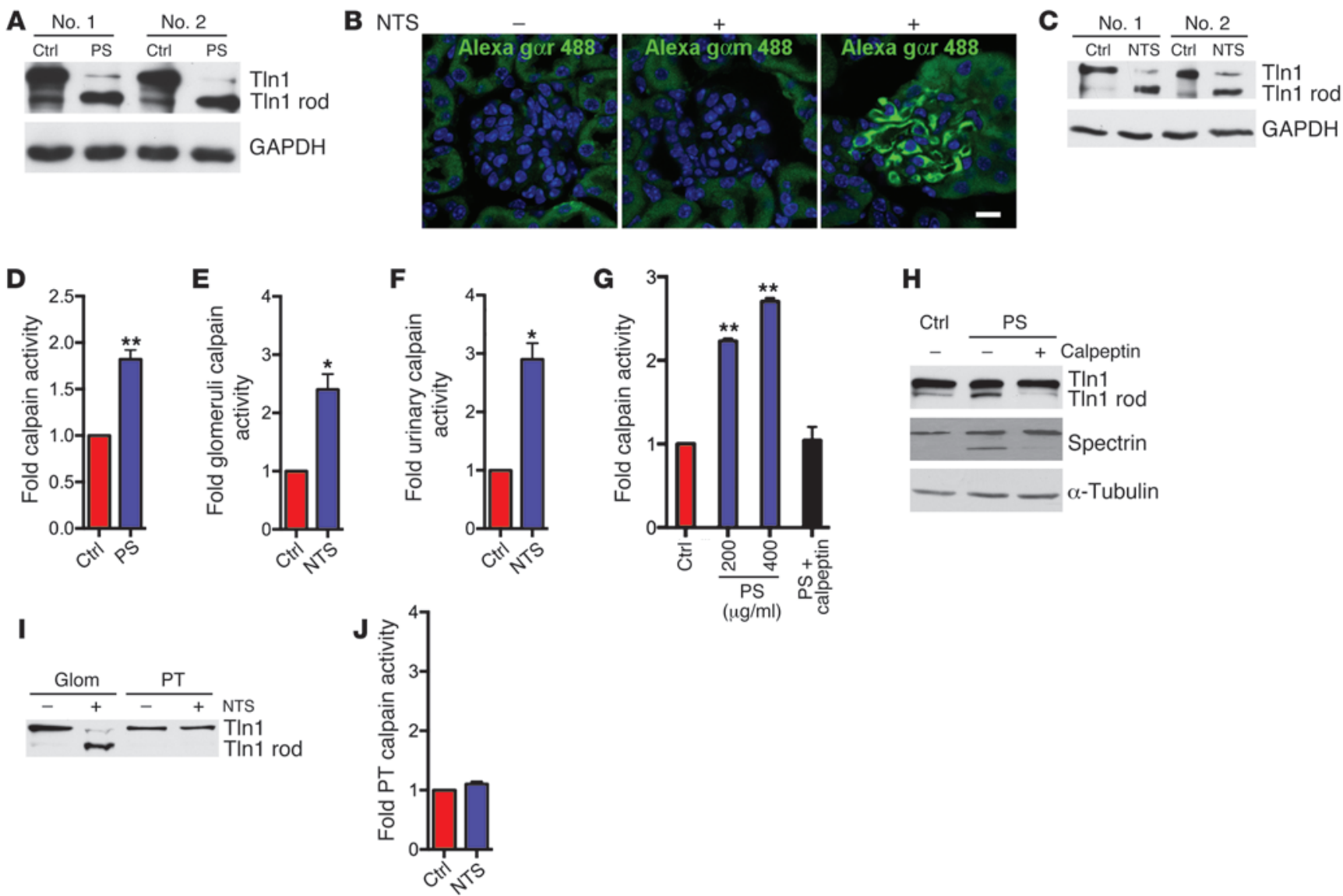

\section{Figure 8}

Calpain-induced talin1 cleavage in injured podocytes. (A) Glomerular lysate isolated following protamine sulfate (PS) infusion into mice demonstrates talin1 cleavage (mouse anti-talin C terminus, clone TD77; Millipore). (B) Injection of NTS in mice demonstrates rabbit IgG deposition in the GBM. Scale bar: $10 \mu \mathrm{m}$. Alexa Fluor goat anti rabbit denoted by gar. Alexa Fluor goat anti-mouse, denoted by gam, demonstrates no signal. (C) Talin1 cleavage is observed in glomeruli following injection of NTS in mice. (D) Glomerular calpain activity is increased following protamine sulfate infusion, $n=7$ experiments. (E) Glomeruli isolated from mice injected with NTS exhibit increased calpain activity, $n=5$ experiments. (F) Increased urinary calpain activity is observed following NTS stimulation. $n=10$ experiments. (G) Supernatant obtained from primary podocytes treated with protamine sulfate exhibit increased calpain activity. $n=3$ experiments. $(\mathbf{H})$ Podocytes stimulated with protamine sulfate $(200 \mu \mathrm{g} / \mathrm{ml})$ demonstrate increased talin1 and spectrin cleavage. (I) Isolated primary proximal tubules show no increase in talin1 cleavage following NTS injection in mice. (J) Isolated primary proximal tubules show no increase in calpain activity following NTS injection in mice. $n=4$. ${ }^{*} P<0.05$; ${ }^{* \star} P<0.01$.

demonstrated a robust talin 1 proteolysis (Figure 8C; quantified in Supplemental Figure 4B). Recent evidence suggests that proteinuric diseases may result from enhanced activity of proteolytic enzymes such as cathespin in podocytes (41). Given that talin1 proteolysis occurs following glomerular injury, we questioned whether this cleavage was due to increased calpain activity $(23,42)$. Glomeruli isolated from mice treated with protamine sulfate or NTS revealed a significant increase in calpain activity when compared with control (Figure 8, D and E). Such pathological differences were present despite equal levels of total calpain as demonstrated by Western blot analysis of both experimental and control glomeruli (data not shown). Moreover, urine samples collected from the NTS-injected mice also revealed an increase in calpain activity (Figure 8F) along with elevated levels of albuminuria (data not shown). To determine whether podocytes were the source of the increased calpain activity, isolated primary podocytes were incubated with or without protamine sulfate. A dose-dependent activation of calpain and concomitant talin 1 cleavage (Figure 8, $\mathrm{G}$ and $\mathrm{H}$ ) was observed, which was abrogated by pretreatment with the calpain inhibitor calpeptin (Figure 8H). Previous reports have suggested that immortalized proximal tubular cells treated with albumin induce calpain activity (43). These results were confirmed by our in vitro cell culture experiments on isolated primary proximal tubular cells treated with BSA at various concentrations (Supplemental Figure 4C). However, to determine whether calpain activity was increased in vivo, primary proximal tubular cells in NTS-treated mice were isolated using a Percoll gradient (validated by $\gamma$ GT enzyme activity and by immunoblotting for proximal tubular marker, megalin; Supplemental Figure 4, D and E). However, the proximal tubules isolated following NTS injection in mice demonstrated neither talin 1 cleavage (Figure 8I) nor increased calpain activity (Figure 8J). This suggests that podocytes may play an important role in calpain secretion and cleavage of talin 1 following injury by NTS. Furthermore, calpain-induced cleavage of other potential substrates, nephrin, FAK, vinculin, dynamin 1 , paxillin, vimentin, and $\beta$-catenin (44-49) in glomeruli, were not observed following infusion of protamine sulfate or injection of NTS (Supplemental Figure 4, F-I). This suggests that cal- 
A
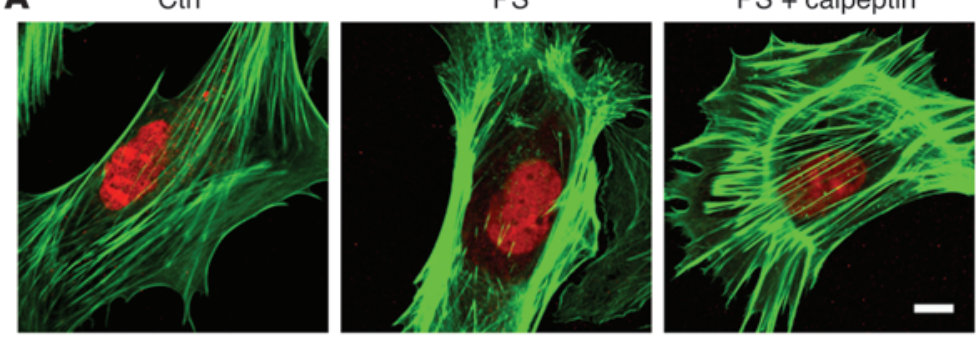

Phalloidin

WT1

C

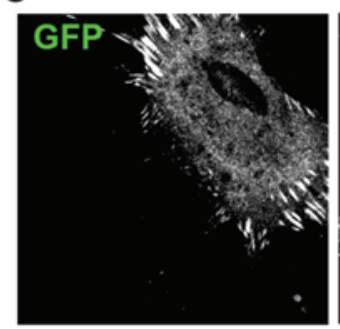

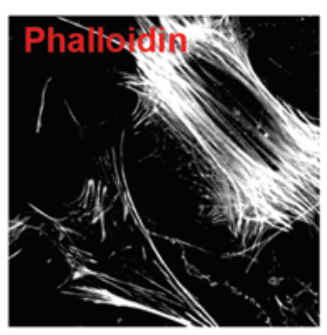

B

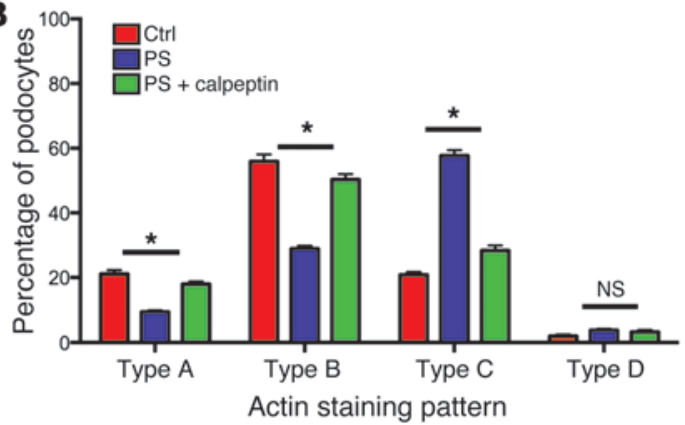

D

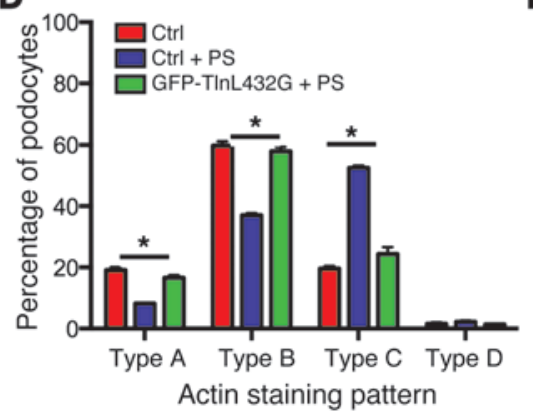

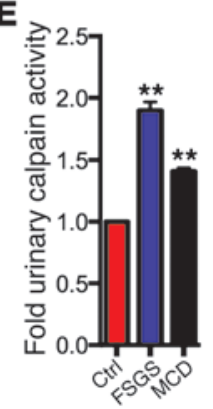

E

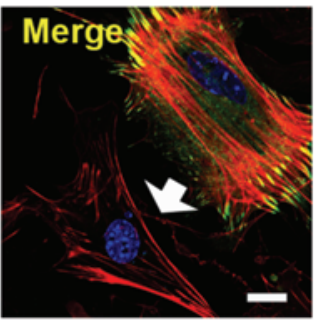

\section{Figure 9}

Increased calpain activity results in actin-cytoskeletal disorganization. (A) Representative image of podocyte phalloidin staining following stimulation with protamine sulfate with or without calpeptin. Scale bar: $10 \mu \mathrm{m}$. (B) Quantification of the phalloidin staining pattern. $n=4$ experiments. ${ }^{*} P<0.05$, control or protamine sulfate plus calpeptin vs. protamine sulfate alone. (C) Representative image of primary podocytes expressing the calpain-resistant talin1 mutant (GFP-Talin L432G) stained with phalloidin following stimulation with protamine sulfate. Note loss of stress fiber in neighboring untransfected WT control (arrow). Scale bar: $10 \mu \mathrm{m}$.(D) Quantification of phalloidin staining pattern of C. $n=3$ experiments. (E) Increased urinary calpain activity is observed in patients with biopsy-proven FSGS and MCD when compared with healthy controls. $n=17$ patients with FSGS, 15 patients with MCD, and 6 healthy controls. ${ }^{*} P<0.05$; ${ }^{* \star} P<0.01$.

pain-induced cleavage of talin 1 is likely critical in the pathogenesis of proteinuria, although other potential calpain substrates may also participate in this process. To determine the potential effect of calpain-induced talin 1 cleavage on the podocyte actin architecture, phalloidin staining was performed on podocytes incubated with protamine sulfate. Actin rearrangement along with the loss of stress fiber formation was observed (Figure 9A; quantified in Figure 9B). However, expression of a calpain cleavage-resistant talin 1 mutant (talin $\mathrm{L} 432 \mathrm{G}$ ) in primary podocytes resulted in the maintenance of stress fibers following treatment with protamine sulfate (Figure 9C; quantified in Figure 9D). This result highlights the importance of calpain-mediated talin 1 cleavage as one of the key determinants of the actin cytoarchitecture in podocytes. To better understand whether alterations in calpain activity might play a role in the pathophysiology of human glomerular disease, we turned our attention to human subjects with biopsy-proven glomerular disease FSGS or MCD. Similar to what was observed in our mouse models of glomerular injury, a robust increase in calpain activity was noted in urine samples from patients with FSGS or MCD when compared with control (Figure 9E).

Inhibiting calpain activity reduces glomerular injury and talin 1 cleavage. To examine the role of the NTS-induced enhancement of calpain activity in the glomerulus, we next determined the expression of the endogenous calpain inhibitor calpastatin. A significant reduction of calpastatin expression in the glomerulus was observed following NTS injection (Figure 10A), which was not seen in the proximal tubules (Supplemental Figure 4J). As the increase in calpain activity may partly be due to the decrease in the expression of the endogenous inhibitor, we next sought to determine the difference in severity of glomerular injury by inhibiting calpain activity exogenously. Mice treated with calpain inhibitor at the time of NTS injection showed a significant reduction in calpain activity (Figure 10B) and talin 1 cleavage in the glomerulus (Figure 10C; quantified in Figure 10D). Furthermore, urine analysis also demonstrated a significant reduction in albuminuria in mice that were treated with calpain inhibitor (Figure 10E). In addition, the evaluation of the podocyte ultrastructure by EM revealed a reduction in podocyte foot process effacement (Figure 10F; quantified in Figure 10G). Lastly, we returned to our Pod-Tln1-KO mice to evaluate whether calpain activity was increased in these nephrotic mice. We also found a significant increase in calpain activity in the urine and glomerulus (Figure 10, H and I). However, inhibiting calpain activity by treatment of the Pod-Tln1-KO mice with calpain inhibitor for 3 weeks failed to rescue the albuminuria in the mutant mice (Figure 10J) These results are consistent with talin 1 being a key substrate of calpain following glomerular injury and with talin1 cleavage in the glomerulus likely playing a pivotal role in disrupting the integrity of the glomerular filtration barrier.

\section{Discussion}

In this study, we demonstrate the importance of talin 1 in both the development and maintenance of the glomerular filtration bar- 

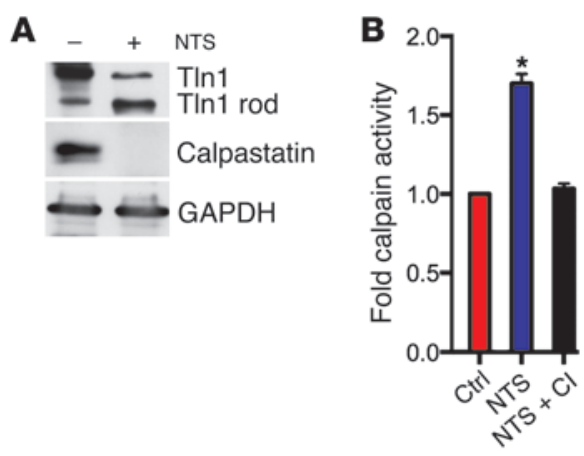
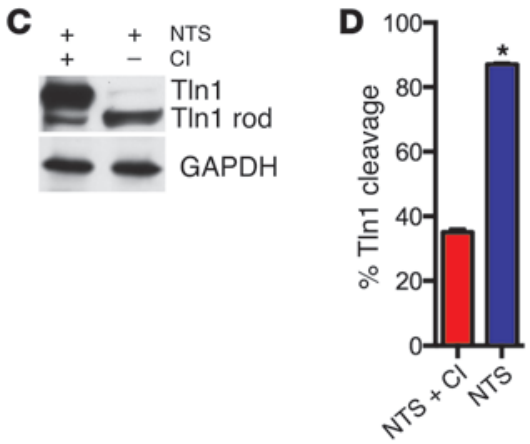

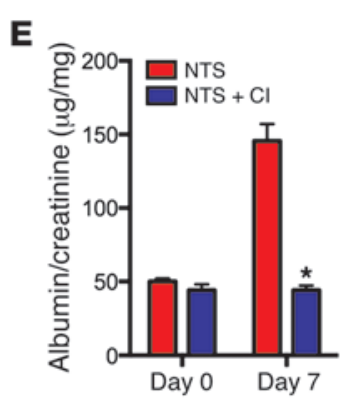

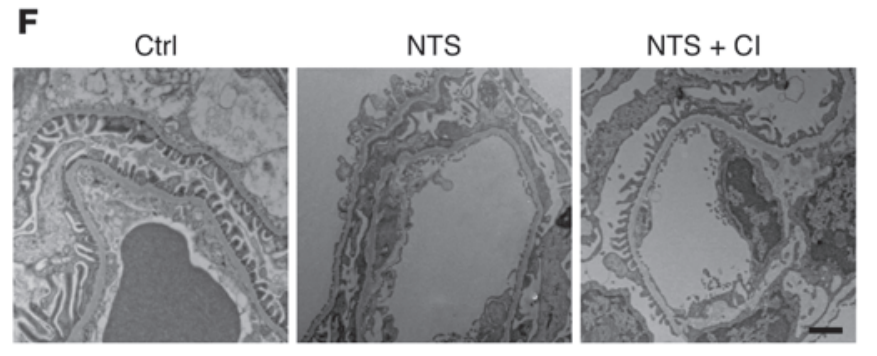
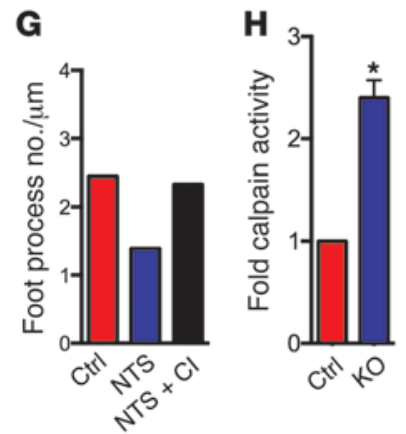
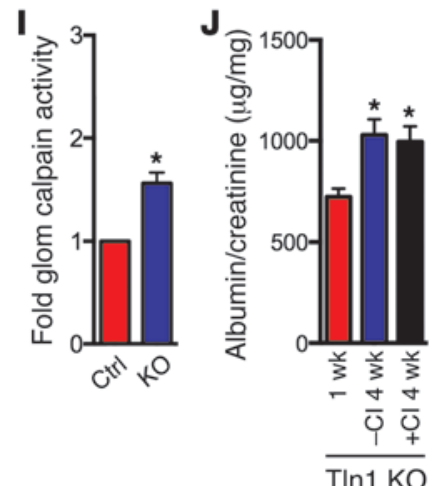

Figure 10

Treatment with calpain inhibitor reduces talin1 cleavage and glomerular injury following NTS treatment. (A) NTS injection in mice reduces calpastatin expression in podocytes. (B) Glomerulus isolated at day 7 following NTS-injected mice treatment with calpain inhibitor (CI) reduces calpain activity. (C) Talin1 cleavage is prevented in NTS-injected mice treated with calpain inhibitor at day 7 after treatment. (D) Quantification of talin1 cleavage in C. (E) Albuminuria is reduced in mice treated with calpain inhibitor at day 7 following NTS injection. (F) Transmission electron micrographs illustrating reduced foot process effacement at 7 days in mice treated with calpain inhibitor following NTS injection. Scale bar: $1 \mu \mathrm{m}$. (G) Quantification of F. (H). Urine isolated from Pod-TIn1-KO mice exhibits increased calpain activity. $n=4$ experiments. (I) Glomeruli isolated from Pod-TIn1-KO mice exhibit increased calpain activity. $n=4$ experiments. (J) Albuminuria is not reduced in Pod-TIn1-KO mice following treatment with calpain inhibitor for 3 weeks. $n=4$ mice. ${ }^{*} P<0.05$.

rier and report that talin 1 is cleaved by calpain following podocyte injury resulting in ne phrotic syndrome. Precisely how talin 1 contributes to the formation and maintenance of podocyte foot processes, a key element of the permeability barrier in kidney, remains to be established. Given that talin 1 activates integrins (50) and is a key link between integrins and the actin cytoskeleton, one plausible mechanism is that talin 1 is required for integrin-dependent tight adhesion of podocytes to the basement membrane, allowing them to withstand the glomerular transcapillary filtration pressure. Furthermore, the loss of tetraspanin CD151, which associates with $\alpha_{3} \beta_{1}$ integrin, has been demonstrated to be involved in strengthening of adhesion to laminin by $\alpha_{3} \beta_{1}$ integrin (51). Consistent with this idea, approximately $15 \%$ of the glomeruli in Pod-Tln1-KO mice have dilated capillary loops similar to those observed in podocytespecific $\beta_{1}$ integrin $\mathrm{KO}$ mice (2). However, our results on podocytes lacking talin 1 expression compared with littermate controls demonstrate only a mild decrease in adhesion and $\beta_{1}$ integrin activation when compared with littermate controls. Furthermore, there was no obvious change in the total podocyte number in kidneys from the Pod-Tln1-KO mice, which is not consistent with the inability of podocytes to adhere tightly to the GBM. Another conceivable explanation is that the loss of talin 1 in podocytes results in a modest reduction in cell spreading, thus preventing the podocytes from tightly covering the glomerular capillaries. However, previous results in mice specifically lacking focal adhesion proteins, FAK, or Crk in podocytes revealed no evidence of proteinuria, even though cell spreading was compromised $(40,52)$.

Interestingly, the phenotype of the Pod-Tln1 Cre mice was significantly more severe than that of mice lacking podocyte $\beta_{1}$ integrin expression (2), with worse glomerulosclerosis, mesangiolysis, and biochemical and histological evidence of end-stage kidney disease. This suggests that much of the phenotype observed in the Pod-Tln1KO mice may possibly be independent of that caused by its alterations of integrin-dependent functions. An alternative explanation for the differences in the phenotype is that it is due to the potential difference in efficiency of the podocin transgene for the excision of integrin $\beta_{1}$ and talin1. Furthermore, differences in the half-life of the 2 proteins following excision may not be identical, accounting for further differences. Loss of talin 1 also has the potential to disrupt the activation and cytoskeletal linkage of other integrins such as $\alpha_{V} \beta_{3}$ in addition to $\alpha_{3} \beta_{1}$ (12). Indeed, there was a dramatic and defective organization of the F-actin cytoskeleton in Pod-Tln1$\mathrm{KO}$ podocytes that could only be rescued by expressing full-length talin1, further demonstrating that the intact talin 1 head and rod play a critical role in maintaining the integrity of the glomerular filtration barrier. Yet podocytes isolated in culture do have imperfect foot processes and slit diaphragms, resulting in a caveat that in vitro mechanistic results may not always mimic in vivo findings. 
It has been demonstrated that mice expressing calpastatin, an endogenous inhibitor of calpain, exhibit reduced proteinuria following NTS injection (43), although the relevant proteolytic substrates remained elusive. Upon examination of talin 1 following podocyte injury by either protamine sulfate or NTS, we noted a robust increase in talin 1 cleavage between the head and rod regions, which was likely due to elevated calpain activity in podocytes, which was not observed in proximal tubules in vivo. Consistent with this, urine analysis in both mouse models of podocyte injury revealed elevated calpain activity, and these findings were further validated in urine samples from patients with nephrotic syndrome (FSGS and MCD). However, we do acknowledge that other cells within the kidney may also secrete calpain following glomerular injury. Calpain-mediated talin cleavage between the head and rod domains is important in focal adhesion turnover and cell migration (23), as is the cleavage of the C-terminal dimerization domain (42). Therefore, degradation of talin 1 mediated by elevated calpain activity observed in podocyte injury and nephrotic syndromes may destabilize podocyte cell-matrix junctions by disrupting the connection between integrins and actin and so contribute to the pathobiology of disease. Indeed, podocytes expressing a calpain-resistant mutant of talin 1 maintained numerous actin stress fibers after treatment with protamine sulfate, unlike the WT controls. Furthermore, mice treated with calpain inhibitor markedly reduced NTS-induced talin 1 cleavage and subsequent proteinuria and foot process effacement.

In summary, we provide evidence that talin 1 is critical for the integrity of the glomerular filtration barrier and demonstrate that calpain-mediated talin 1 cleavage may play a critical role in the pathology resulting following podocyte injury. These data suggest that either deleting talin 1 or inducing talin 1 cleavage by calpain decouples the intimate link between integrins at the GBM and the actin cytoskeleton. Calpain-mediated cleavage of additional focal adhesion components such as FAK, vinculin, or paxillin was not observed following NTS or protamine sulfate infusion. The exact mechanism of how calpain activity is increased in podocytes following injury requires further investigation. We have shown that following injury, calpastatin, the endogenous inhibitor of calpain, is remarkably reduced in the glomerulus. It has also been demonstrated that calpain 2 can be increased following Erk phosphorylation of serine 50. Further corroborating this possibility, recent evidence suggests that Erk phosphorylation is increased following glomerular injury (53). These results further emphasize the critical role of podocyte cell-matrix interactions in glomerular function in mice $(2,4)$, and humans $(24,25)$ and how therapeutic targeting of these pathways may benefit patients (54). Furthermore, it highlights the importance of future studies on other integrin-associated proteins such as kindlin, which can interact with integrins, phosphoinositides, and ILK (55). Collectively, these findings highlight the importance of developing a greater understanding of cellmatrix interactions as it relates to nephrotic syndromes and shed light on what we believe are novel therapeutic strategies, such as the potential blockade of calpain activity following glomerular injury.

\section{Methods}

$A b s$, reagents, and expression constructs. Abs used in this study were as follows: mouse anti-talin1 $(T \ln 1)$ clone $97 \mathrm{H} 6$ and mouse anti-talin2 $(T \ln 2)$ clone 68E7 (56), available from Cancer Research Technology (talin1, http://www. cancertechnology.co.uk/talin-1-0; talin2, http://www.cancertechnology. co.uk/talin-2), mouse anti-talin (C-terminal) clone TD77 (Millipore), rat anti-integrin $\beta_{1}$ clone MB1.2 (Chemicon), rat anti-CD29 clone 9EG7 (BD Biosciences), mouse anti-FAK clone 77/FAK (BD Biosciences), mouse antipaxillin clone 165/paxillin (BD Biosciences), rabbit anti-ILK (Cell Signaling), rabbit anti-integrin $\alpha 3$ (Millipore), rabbit anti-WT1 (Santa Cruz Biotechnology Inc.), mouse anti-vinculin clone hVIN-1 (Sigma-Aldrich), rabbit anti-calpastatin (Thermo Scientific), mouse anti- $\beta$ catenin (Sigma-Aldrich), rabbit anti-dynamin1 clone EP801Y (Epitomics), mouse anti-Vimentin (BD Biosciences), mouse anti-GFP clone 7.1 and 13.1 (57), mouse anti- $\beta$-actin clone 6G3, and mouse anti-GAPDH clone 1C4 (Sangene Biotech). Alexa Fluor 488 goat anti-rabbit IgG Ab, Alexa Fluor 488 goat anti-rat IgG Ab, Alexa Fluor 488 goat anti-mouse IgG Ab, Alexa Fluor 594 goat anti-rabbit IgG Ab, Alexa Fluor 594 goat anti-mouse IgG Ab, and Alexa Fluor 488-conjugated phalloidin were purchased from Invitrogen. Rabbit anti-nephrin Ab was a gift from Yutaka Harita (University of Tokyo, Tokyo, Japan) (58). Rabbit anti-megalin was a gift from Daniel Biemesderfer (Yale University) (59). Plasmids encoding enhanced GFP-Tln1 (EGFP-Tln1) full-length plasmid (residues 1-2541) and EGFP-Tln1 head domain (residues 1-435) plasmid were provided by David Calderwood (Yale University) (60). EGFP-Tln1 rod domain plasmid (residues 435-2541) was a gift from Anna Huttenlocher (University of Wisconsin, Madison, Wisconsin, USA) (61). CD8-rat nephrin-Flag (pCMV-tag4; Stratagene) construct was provided by Yutaka Harita (University of Tokyo) (58). mCherry-Utrophin was provided by William Bement (University of Wisconsin) (39). EGFP-Th1 L432G was obtained from Addgene. Cell culture media was purchased from Invitrogen. Collagen type I, laminin, and fibronectin were purchased from BD Biosciences. Protamine sulfate was purchased from Sigma-Aldrich. ChromPure rabbit IgG and CFA were purchased from Jackson Immunoresearch Laboratories and Sigma-Aldrich, respectively. Calpeptin, fluorogenic calpain substrate (Succinyl-Leu-Tyr-AMC, where AMC indicates 7-amino-4-methylcoumarin), and AMC were purchased from Calbiochem. Calpain inhibitor III for mouse experiments was purchased from Bachem.

Creation and genotyping of podocyte-specific Th1-KO mice. For selective deletion of $T \ln 1$ in glomerular podocytes, $T \ln 1^{f / f l}$ mice (62) were crossed with Podocin-Cre mice (32) to generate a podocyte-specific KO of Tln1 (Pod-Tln1$\mathrm{KO})$. Tail genotyping was performed by PCR using previously described protocols $(32,63)$. Pod-Tln1-KO mice (homozygous for the floxed Tln 1 allele with Podocin-Cre) and littermate Tln $1^{f / / f l}$ controls (homozygous for the $T \ln 1$ allele but lacking Podocin-Cre) were used in these experiments.

Biochemical measurements: plasma creatinine, urine albumin, and urine creatinine. Urine samples were collected from the Pod-Th1-KO and littermate $T \ln 1^{f / f l}$ controls. Albuminuria was qualitatively assessed by $10 \%$ SDS-PAGE followed by Coomassie blue staining. Urine albumin levels were measured qualitatively and in duplicate using an albumin ELISA quantitation kit according to the manufacturer's protocol (Bethyl Laboratories Inc.), and the absorbance read at $450 \mathrm{~nm}$ (glomax multi detection system; Promega) as previously described $(38,52)$. Urine and plasma creatinine were measured in duplicate for each sample with an ELISA quantitation kit (Bioassay Systems) at an absorbance of $490 \mathrm{~nm}$ (Bio-Rad Microplate Reader).

Cell culture and transfection. Isolation of podocytes from P1-P3 Pod-Th1$\mathrm{KO}$ and $T \ln 1^{f / f l}$ pups was performed as described previously in our laboratory $(38,52)$. Briefly, mouse glomeruli were harvested using Dynabead (Invitrogen) perfusion' the enriched glomeruli were passed through a $100-\mu \mathrm{m}$ cell strainer (Falcon; BD Biosciences) and plated on collagen type Icoated dishes at $37^{\circ} \mathrm{C}$ in RPMI 1640 medium with 9\% FBS, $100 \mathrm{U} / \mathrm{ml}$ penicillin, $100 \mu \mathrm{g} / \mathrm{ml}$ streptomycin, $100 \mathrm{mM}$ HEPES, $1 \mathrm{mM}$ sodium bicarbonate, and $1 \mathrm{mM}$ sodium pyruvate. Subculture of primary podocytes was performed by detaching the glomerular cells with $0.25 \%$ trypsin-EDTA (Invitrogen), followed by sieving through a $40-\mu \mathrm{m}$ cell strainer (Falcon; BD Biosciences), and culture on collagen type I-coated dishes. Podocytes of passages 1 or 2 were used in all experiments. 
Transfections were performed by electroporation with the Gene Pulser Electroporation System (Bio-Rad) according to the manufacturer's instructions, using $4 \mu \mathrm{g}$ of DNA. The transfected $P o d-T \ln 1-\mathrm{KO}$ and $T \ln 11^{f / / f l}$ primary podocytes were plated on collagen type I-coated coverslips and were analyzed as specified in each experiment after transfection for 48 hours.

Cell culture and shRNA. Mouse $T \ln 1$-specific shRNAs encoded in a PGIPZ vector were purchased from Open Biosystems; sequences were as follows: 1: 5'-TGCTGTTGACAGTGAGCGCGCAGCCACTATTGTAGCCAAATAGTGAAGCCACAGATGTA-3'; 2: 5'-TGCTGTTGACAGTGAGCGCCAGGATGGCTACTACTCAGTATAGTGAAGCCACAGATGTA-3'). Tln 1 shRNA oligonucleotides were transfected together with 2 lentiviral packaging vectors, PCMVAR8.91 and PMD.G, into 293T cells with lipofectamine 2000 in Opti-MEM (Invitrogen) as previously described (52). The supernatant was filtered $(0.45 \mu \mathrm{m})$, and the $T \ln 1^{f / f l}$ primary podocytes at subconfluency were infected twice with the filtered supernatant. A scramble shRNAmir (Open Biosystems) served as a negative control.

Western blot. Freshly isolated glomeruli, cultured primary podocytes, and proximal tubules were lysed into lysis buffer containing $50 \mathrm{mM}$ Tris- $\mathrm{HCl}$ ( $\mathrm{pH}$ 7.6), $500 \mathrm{mM} \mathrm{NaCl}, 0.1 \%$ SDS, $0.5 \%$ deoxycholate, $1 \%$ Triton $\mathrm{X}-100$, $0.5 \mathrm{mM} \mathrm{MgCl}_{2}$, and protease inhibitor cocktail (Roche Diagnostics). After determination of protein concentrations by the Bio-Rad Protein Assay, equal amounts of total protein were denatured for 10 minutes at $95^{\circ} \mathrm{C}$, resolved 4\%-20\% gradient SDS-PAGE gels, and transferred to the Immobilon PVDF membranes (Millipore). The membrane was blocked with 5\% nonfat milk in $1 \times$ TBST and incubated with the appropriate primary $\mathrm{Ab}$ at $4{ }^{\circ} \mathrm{C}$ overnight. Following 3 washes with $1 \times$ TBST, the appropriate peroxidase-labeled anti-IgG secondary Ab (Bio-Rad) was added and signals detected using enhanced chemiluminescence reagents (PerkinElmer).

Detection of talin 1 cleavage. Primary podocytes isolated from $T \ln 1^{f l / f l}$ mice were incubated with or without calpeptin or DMSO for 30 minutes prior to stimulation with protamine sulfate for 1 hour at $37^{\circ} \mathrm{C}$. The cell lysates were used for analysis of talin 1 protein expression by immunoblotting with talin $1 \mathrm{Ab}$, and the cells were used for actin-staining pattern analysis by immunofluorescence.

Animal perfusions with protamine sulfate were carried out as previously described (40). Briefly, 3-month-old $T \ln 1^{f l / f l}$ mice were anesthetized with $1 \%$ ketamine and $0.1 \%$ xylazine $(0.1 \mathrm{ml} / 10 \mathrm{~g}$ body weight $)$ and animals maintained at $37^{\circ} \mathrm{C}$ throughout the procedure using a surgical heating pad apparatus. Kidneys were perfused with solutions maintained at $37^{\circ} \mathrm{C}$ through the left ventricle at a pressure of approximately $70 \mathrm{mmHg}$ and an infusion rate of $10 \mathrm{ml} / \mathrm{min}$. Perfusion was carried out with HBSS for 2 minutes followed by perfusion with protamine sulfate $(2 \mathrm{mg} / \mathrm{ml}$ in HBSS) for 15 minutes. Glomeruli were harvested and enriched from mice kidney cortical tissue with $45 \%$ Percoll solution (GE Healthcare) as previously described (64). Briefly, the kidneys were immediately harvested and washed in ice-cold Krebs-Henseleit saline solution (KHS) $(119 \mathrm{mM} \mathrm{NaCl}$, $4.7 \mathrm{mM} \mathrm{KCl}, 1.9 \mathrm{mM} \mathrm{CaCl}_{2}, 1.2 \mathrm{mM} \mathrm{KH}_{2} \mathrm{PO}_{4}, 1.2 \mathrm{mM} \mathrm{MgSO} \cdot 7 \mathrm{H}_{2} \mathrm{O}$, and $25 \mathrm{mM} \mathrm{NaHCO}_{3}, \mathrm{pH}$ 7.4). The renal cortex segments were dissected away and finely minced into small pieces and incubated with $1 \mathrm{mg} / \mathrm{ml}$ collagenase (Roche) at $37^{\circ} \mathrm{C}$ for 30 minutes. The solution was filtered through a $100-\mu \mathrm{m}$ sieve (BD Falcon), and the filtrate was centrifuged at $600 \mathrm{~g}$ for 5 minutes at $4{ }^{\circ} \mathrm{C}$ and then washed 3 times in KHS. The final pellet was resuspended in $30 \mathrm{ml}$ of $45 \%$ Percoll solution (GE Healthcare Bio-Sciences) and centrifuged (Sorvall model: SA-600) at 20,000 $\mathrm{g}$ for 45 minutes at $4^{\circ} \mathrm{C}$. Centrifugation resulted in the separation of 4 banded layers. The solution within the first and third layers resulted in the enrichment of the glomerulus and proximal tubules, respectively, and following aspiration, was centrifuged at $600 \mathrm{~g}$ for 5 minutes at $4{ }^{\circ} \mathrm{C}$ to remove the Percoll solution. The activity of brush border enzyme for $\gamma$-glutamyl transpeptidase ( $\gamma \mathrm{GT}$ ) (in proximal tubules) and the protein expression of neph- rin (in glomerulus) and megalin (in proximal tubules) were performed to confirm purity. The glomerular cell lysates were used for analysis by immunoblotting with the talin $1 \mathrm{Ab}$.

For mice treated with NTS generated by Lampire Biological Laboratories, experiments were performed as previously described in our laboratory (52). Briefly, mice were preimmunized 3 days prior to administration of NT, via intraperitoneal injection of $250 \mu \mathrm{g}$ of rabbit IgG (Jackson Immunoresearch Laboratories) in $250 \mu$ l of 1:1 emulsion with CFA (Sigma-Aldrich). Glomerulonephritis was induced with $200 \mu \mathrm{l}$ of NTS as previously described (52). Preimmune rabbit serum was used as a negative control. Calpain activity was determined in urine samples obtained on days 0 and 1 and evaluated by calpain activity assay. Mice were anesthetized, and kidneys were collected for determination of the calpain activity from isolated glomeruli on day 1 . The glomeruli were harvested and processed for Western blot and kidney tissues were harvested and processed for immunofluorescence. For calpain inhibitor studies, calpain inhibitor III was injected once daily for 7 days intraperitoneally ( $30 \mathrm{mg} / \mathrm{kg}$ body weight) starting the same day as NTS injection. Control mice received an equal volume of PEG400/DMSO $(65,66)$. Mice urine samples were obtained on days 0 and 7 and were evaluated for calpain activity following NTS injection. On day 7, mice were anesthetized and kidneys were collected for determination of calpain activity and talin 1 cleavage in the isolated glomeruli. Kidney sections were also processed for EM. For talin1 cleavage quantification, densitometry was performed by NIH ImageJ software and expressed by dividing the talin1rod cleavage product by the sum of the talin 1 plus talin1-rod densitometry.

Culture of mice renal proximal tubules. For cultured proximal tubules isolated from the Percoll solution as described, cells were seeded in DMEM and, following $75 \%$ confluency, serum starved overnight; this was followed by administration of BSA $(0 \mathrm{mg} / \mathrm{ml}, 2.5 \mathrm{mg} / \mathrm{ml}, 5 \mathrm{mg} / \mathrm{ml}, 10 \mathrm{mg} / \mathrm{ml}$, $20 \mathrm{mg} / \mathrm{ml}$ ) for 6 hours at $37^{\circ} \mathrm{C}$. The extracellular calpain activity was determined by measurement of the calpain-specific cleavage of fluorescent AMC obtained from the cell supernatants.

Kidney bistology, immunofluorescence staining of kidney tissues, and quantification. Mice were anesthetized by intraperitoneal injection of ketamine followed by perfusion fixation with $40 \mathrm{ml}$ of $4 \%$ paraformaldehyde with or without $2 \%$ glutaraldehyde for immunofluorescence or EM experiments, respectively. For histology, kidney sections were sent to the Yale Pathology Core Tissue Services for H\&E, PAS, or Masson's trichrome (TRI) staining. For transmission EM, kidneys were post-fixed with Palade's osmium (4\% OSO4 in double-distilled water, $\mathrm{pH}$ 7.6). EM was performed by the Cellular and Molecular Physiology Core Services at Yale University. For scanning EM, tissues were post-fixed with osmium in $0.1 \mathrm{M}$ sodium cacodylate and $0.1 \mathrm{M}$ sucrose, $\mathrm{pH} 7.4$, and dehydrated through an ethanol series. Samples were critical-point-dried with $\mathrm{CO}_{2}$ as the transitional fluid, sputter-coated with gold-palladium, and examined with an ISI SS40 SEM at $10 \mathrm{kV}$ (Yale University). For Immunogold labeling, mice were anesthetized by intraperitoneal injection of ketamine followed by perfusion with $3 \%$ paraformaldehyde and $0.01 \%$ glutaraldehyde in $100 \mathrm{mM}$ Cacodylate. Ultrathin kidney sections were quenched with $0.5 \mathrm{M}$ ammonium chloride/ TBS and incubated with the mouse anti-talin 1 clone 97H6, followed by gold-conjugated secondary $\mathrm{Ab}$.

For immunofluorescence, kidney cryosections were subjected to antigen retrieval at $95^{\circ} \mathrm{C}$ for 10 minutes in Retrievagen A solution ( $\mathrm{pH}$ 6.0) (BD Biosciences), followed by blocking with $3 \%$ BSA in $1 \times$ PBS for 1 hour at room temperature (RT). Immunostaining of cryosections was performed as follows: slides were incubated with the appropriate primary Abs overnight at $4{ }^{\circ} \mathrm{C}$ followed by incubation with Alexa Fluor 488 and/or 594-conjugated secondary Abs at RT for 1 hour and washed three times with $1 \times$ PBS; then coverslips were mounted using Vecta Shield containing 4,6-diamidino-2-phenyllinodole (Vector Laboratories). Images were 
acquired with the Zeiss LSM 710 laser scanning confocal microscope using a $\times 63$ Plan Apo $(\mathrm{NA}=1.4)$ oil immersion objective for immunofluorescence analysis, and images were processed using NIH ImageJ software or Adobe Photoshop CS4.

For quantitative analysis of kidney histology, 50 full-sized glomeruli for each specimen were assessed on PAS-stained sections, and the level of glomerulosclerosis in each glomerulus was semiquantitatively scored as follows: 0 , no sclerosis; 1 , sclerosis; less than $10 \%$ of glomeruli; 2 , sclerosis $10 \%$ to approximately $25 \%$ of glomeruli; 3 , sclerosis $25 \%$ to approximately $50 \%$ of glomeruli; 4 , sclerosis more than $50 \%$ of glomeruli. To evaluate interstitial fibrosis, 20 fields for each section were assessed on TRI-stained sections. Semiquantitative analysis in each field was assessed as follows: 0 , no fibrosis; 1 , fibrosis less than $10 \%$ of areas; 2 , fibrosis $10 \%$ to approximately $25 \%$ of areas; 3 , fibrosis $25 \%$ to approximately $50 \%$ of areas; 4 , fibrosis more than $50 \%$ of areas. The averages of the glomerulosclerosis and interstitial fibrosis scores were calculated from the total evaluated glomeruli or interstitial lesions in each section. These microscopic evaluations were performed by Sung Hyun Son, without prior prejudicial information.

For quantitative ultrastructural analysis of the glomerulus by transmission EM, the number of podocyte foot processes present in each micrograph was divided by the total length of GBM regions in each image to determine the average density of podocyte foot processes. The GBM thickness in each image was also measured by NIH ImageJ software or Adobe Photoshop software.

Immunofluorescence staining of primary podocytes and quantification of actin stress fibers and focal adhesions. Cultured isolated primary podocytes were fixed in $4 \%$ paraformaldehyde in $1 \times$ PBS, permeabilized with $0.1 \%$ Triton $\mathrm{X}-100$ in $1 \times$ PBS for 10 minutes, blocked with $3 \%$ BSA, then incubated with the appropriate primary and secondary Abs as described above. Images were acquired and processed as described above.

For quantitative analysis of changes in the actin cytoarchitecture of primary podocytes, the multiplicity of different phalloidin staining patterns was grouped into 4 major classes and used for scoring as follows: type A: more than $90 \%$ of cell area filled with thick cables; type B: at least 2 thick cables running under nucleus and rest of cell area filled with fine cables; type C: no thick cables, but some cables present; type D: no cables visible in the central area of the cell (67). Before scoring, labels on all slides were blind-coded by someone other than scorer. One hundred cells in each slide were analyzed in 3 separate experiments.

The quantification of focal adhesion numbers was performed using immunofluorescence stained for FAK and paxillin on stably adherent cells. The number of focal adhesions and the podocyte cell area were manually traced along the cell perimeter using ImageJ software. The total numbers of focal adhesions were then normalized to the corresponding area of the cell, and the data were reported as number per $1000 \mu \mathrm{m}^{2}$ of cell area (68). Twenty-five cells were analyzed in each experiment for control and PodTln1-KO podocytes, with 3 experiments performed.

Live cell imaging and analysis. Live cell imaging was performed as described previously (69). Briefly, mCherry-Utrophin was coexpressed in primary podocytes by electroporation (Amaxa Nucleofector Kit R). Transfected cells were seeded in glass-bottomed $35-\mathrm{mm}$ culture dishes (no. 1.5 thickness) (MatTek) and imaged 24 hours later. Before imaging, medium was replaced with an imaging buffer (containing $136 \mathrm{mM} \mathrm{NaCl}, 2.5 \mathrm{mM} \mathrm{KCl}, 2 \mathrm{mM}$ $\mathrm{CaCl}_{2}, 1.3 \mathrm{mM} \mathrm{MgCl}_{2}$, and $10 \mathrm{mM}$ Hepes at $\mathrm{pH}$ 7.4). Cells were imaged using the PerkinElmer spinning confocal microscope as previously described (38).

Adhesion assay. Adhesion assays with crystal violet staining were performed as previously described (36). Briefly, primary podocytes from Pod$\operatorname{Tln} 1-\mathrm{KO}$ and $T \ln 1^{f / f l}$ control mice were trypsinized and seeded on 96-well plates coated with collagen type $\mathrm{I}(10 \mathrm{mg} / \mathrm{ml})$, laminin $(10 \mathrm{mg} / \mathrm{ml})$, or fibronectin $(10 \mathrm{mg} / \mathrm{ml})$ at a density of $1.5 \times 10^{4}$ cells per well. After 2 hours incubation at $37^{\circ} \mathrm{C}$, nonadherent cells were removed by gentle washing with $1 \times$ PBS, followed by fixation in $95 \%$ ethanol. Cells were stained in $0.1 \%$ crystal violet (Sigma-Aldrich) for 15 minutes at RT, washed in water, and lysed in $1 \%$ SDS while shaking until uniform color was obtained. Absorbance at $595 \mathrm{~nm}$ was measured using a microplate reader (Bio-Rad Model 550). Four or five independent experiments were performed.

Spreading assays. Cell spreading was determined using live imaging by phase-contrast microscopy using a Nikon Eclipse TE200 equipped with a $\times 20$ objective, motorized scanning table, and a stage incubator at $37^{\circ} \mathrm{C}$ in $5 \% \mathrm{CO}_{2}$. Images were captured with Hoffman modulation and Spot RT camera (Diagnostic Instruments). Podocytes of Pod-Thl-KO and $T \ln 11^{f / f l}$ controls were seeded on a laminin-coated $35 \times 10 \mathrm{~mm}$ tissue culture dish, and image acquisition was started immediately. Images were collected every 5 minutes for 2 hours. Cell area over time was analyzed using NIH Image software in a blinded manner by randomly examining 25 cells per genotype for each experiment. Eight independent experiments were performed.

CD8-nephrin chimera endocytosis assay. For internalization studies, the cells expressing CD8-rat nephrin-Flag, were washed with cold PBS and labeled for 1 hour at $4{ }^{\circ} \mathrm{C}$ with mouse anti-CD8 Ab in serum-free RPMI. Unbound $\mathrm{Ab}$ was removed by 3 washes with cold PBS prior to incubating the cells at $37^{\circ} \mathrm{C}$ in prewarmed complete RPMI. After internalization of bound label for varying time intervals, the cells were washed with $\mathrm{PBS}$, and surface-retained mouse anti-CD8 $\mathrm{Ab}$ was labeled at $4^{\circ} \mathrm{C}$ for 40 minutes with Alexa Fluor 488-conjugated anti-mouse Ab in serum-free RPMI medium, followed by extensive washing with cold PBS and fixation with $4 \%$ paraformaldehyde. The cells were then permeabilized with $0.1 \%$ Triton-X 100 and blocked with 5\% BSA in PBS; mouse anti-CD8 Ab was detected with Alexa Fluor 594 anti-mouse Ab. The cells were washed 3 times with PBS, followed by mounting with Vectashield (Vector Laboratories). Images were acquired with a Zeiss LSM 710 laser scanning confocal microscope using a $\times 63$ Plan Ao (NA = 1.4) oil immersion objective. To quantify CD8-nephrin endocytosis, fluorescence intensities of cellsurface-retained nephrin (Alexa Fluor 488 signal originating from the cell surface) and internalized nephrin (Alexa Fluor 594 signal from the cell interior) were measured with NIH ImageJ software by examining 20 cells per experiment $(n=3)$ from each indicated time point. We defined the endocytosed nephrin by subtracting the Alexa Fluor 488 image from the Alexa Fluor 594 image and normalizing to surface-derived fluorescence intensity [(Alexa Fluor 594 - Alexa Fluor 488)/Alexa Fluor 488]. As a negative control, anti-CD8 Ab staining on untransfected cells was evaluated and found to be minimal.

FACS assay. Freshly isolated primary podocytes from Pod-Tln1-KO mice and $T \ln 1^{f / f l}$ controls $\left(5 \times 10^{5}\right.$ cells per sample) were stained with primary Abs against total $\beta_{1}$ integrin (1:200; clone MB1.2; recognizes all $\beta_{1}$ integrins) or activated $\beta_{1}$ integrin (1:100; clone 9EG7; recognizes an activation-associated epitope) in $1 \times$ PBS containing $3 \%$ BSA for 30 minutes on ice, followed by washing and staining with secondary Abs labeled with FITC for an additional 30 minutes on ice. Dead cells were excluded by the addition of $2.5 \mu \mathrm{g} / \mathrm{ml}$ propidium iodide prior to FACS analysis. FACS was conducted on the FACSCalibur flow cytometer (BD Biosciences) and analyzed with FACSCalibur CellQuest Pro Software. Three independent experiments for each genotype were performed.

Calpain activity assay. Calpain activity in the podocyte lysates was determined using a calpain activity assay kit (Abcam) according to the manufacturer's protocol. Briefly, podocyte lysate was incubated with substrate (AcLLY-AFC, where AFC indicates 7-amino-4-trifluoromethyl coumarin) and $\times 10$ reaction buffer from the manufacturer for 1 hour at $37^{\circ} \mathrm{C}$ in the dark. Upon cleavage of substrate by calpain, the fluorogenic portion of AFC was detected at a wavelength of $505 \mathrm{~nm}$ following excitation at $400 \mathrm{~nm}$. Fluorescence emission was measured by a SpectraMax MS Spectrofluo- 
rometer (Molecular Devices). For measuring the calpain activity in urine (concentration normalized by urine creatinine) or isolated glomeruli, a $25 \mu \mathrm{l}$ mouse urine sample or $100 \mu \mathrm{g}$ glomeruli were diluted in KrebsRinger buffer (KRB) solution ( $\mathrm{pH}$ 7.4) supplemented with $2 \mathrm{mM} \mathrm{CaCl}_{2}$ in a 96-well plate. These samples were exposed in the dark at $37^{\circ} \mathrm{C}$ with $50 \mu \mathrm{M} \mathrm{N}$-succinyl-leucine-leucine-valine-tyrosine-AMC (Suc-Leu-LeuVal-Tyr-AMC), a membrane-permeant calpain protease substrate. After a 90-minute incubation period, AMC fluorescence was detected at 365 $\mathrm{nm}$ excitation and $460 \mathrm{~nm}$ emission (Glomax Multi Detection System; Promega). An AMC standard curve (0 to $40 \mu \mathrm{M})$ was generated for each experiment. Each sample was measured in duplicate.

Statistics. Data were expressed as mean \pm SEM. Statistical analysis was performed using 2-tailed Student's $t$ test or 1-way ANOVA (SigmaStat, version 3.1.1). Survival analysis was carried out using the log-rank test (Prism 5; GraphPad). $P<0.05$ was considered significant.

Study approval. All animal experiments were approved by the University Committee on the Use and Care of Animals Institutional Review Board at Yale University. All work was conducted in accordance with the principles and procedures outlined in the NIH Guide for the Care and Use of Laboratory Animals (Revised 2011). All human urine samples analyzed were obtained from biopsy-proven active FSGS and MCD patients or 6 control urine sam- ples, provided by Eduardo Garin (University of Florida, Gainsville, Florida, USA) according to IRB \# 481-2008 after obtaining patient consent.

\section{Acknowledgments}

We are grateful to Pietro De Camilli (Yale University) for access to spinning disc confocal microscopy, and Eduardo Garin at the University of Florida for the patient samples. This work was supported in part from the following sources: NIH DK083294, NIH DK093629 and a Norman Coplon Satellite Healthcare grant (to S. Ishibe); NIH grants and a grant from the George O'Brien Kidney Center at Yale, P30DK079310 (to S. Ishibe); and NIH DK073495, NIH DK089394, and NIH 1R01 DK101350-01 (to J. Reiser). Ramiro Nandez was supported by NIH grant DK082700 to Pietro De Camilli.

Received for publication September 23, 2013, and accepted in revised form December 5, 2013.

Address correspondence to: Shuta Ishibe, 300 Cedar Street, S369C, P.O. Box 208209, New Haven, Connecticut 06520-8029, USA. Phone: 203.737.4170; Fax: 203.785.4904; E-mail: shuta.ishibe@yale.edu.
1. Sachs N, Sonnenberg A. Cell-matrix adhesion of podocytes in physiology and disease. Nat Rev Nephrol. 2013;9(4):200-210.

2. Pozzi A, et al. $\beta 1$ Integrin expression by podocytes is required to maintain glomerular structural integrity. Dev Biol. 2008;316(2):288-301.

3. Kreidberg JA, et al. $\alpha 3 \beta 1$ Integrin has a crucial role in kidney and lung organogenesis. Development. 1996;122(11):3537-3547.

4. Sachs N, et al. Kidney failure in mice lacking the tetraspanin CD151. J Cell Biol. 2006;175(1):33-39.

5. Jarad G, Cunningham J, Shaw AS, Miner JH. Proteinuria precedes podocyte abnormalities in Lamb2 $2^{-/}$ mice, implicating the glomerular basement membrane as an albumin barrier. J Clin Invest. 2006; 116(8):2272-2279.

6. Wei C, et al. Circulating urokinase receptor as a cause of focal segmental glomerulosclerosis. Nat Med. 2011;17(8):952-960.

7. Bouaouina M, Harburger DS, Calderwood DA. Talin and signaling through integrins. Methods Mol Biol. 2012;757:325-347.

8. Dai C, et al. Essential role of integrin-linked kinase in podocyte biology: Bridging the integrin and slit diaphragm signaling. J Am Soc Nephrol. 2006; 17(8):2164-2175

9. El-Aouni $\mathrm{C}$, et al. Podocyte-specific deletion of integrin-linked kinase results in severe glomerular basement membrane alterations and progressive glomerulosclerosis. J Am Soc Nephrol. 2006; 17(5):1334-1344

10. Kanasaki K, et al. Integrin beta1-mediated matrix assembly and signaling are critical for the normal development and function of the kidney glomerulus. Dev Biol. 2008;313(2):584-593.

11. Debrand E, et al. Talin 2 is a large and complex gene encoding multiple transcripts and protein isoforms. FEBS J. 2009;276(6):1610-1628.

12. Critchley DR. Biochemical and structural properties of the integrin-associated cytoskeletal protein talin. Annu Rev Biophys. 2009;38:235-254.

13. Garcia-Alvarez B, et al. Structural determinants of integrin recognition by talin. Mol Cell. 2003; 11(1):49-58.

14. Tadokoro $S$, et al. Talin binding to integrin $\beta$ tails: a final common step in integrin activation. Science. 2003;302(5642):103-106.

15. Di Paolo G, et al. Recruitment and regulation of phosphatidylinositol phosphate kinase type $1 \gamma$ by the FERM domain of talin. Nature. 2002;
420(6911):85-89

16. Barsukov IL, et al. Phosphatidylinositol phosphate kinase type $1 \gamma$ and $\beta 1$-integrin cytoplasmic domain bind to the same region in the talin FERM domain. J Biol Chem. 2003;278(33):31202-31209.

17. Lee SY, Voronov S, Letinic K, Nairn AC, Di Paolo G, De Camilli P. Regulation of the interaction between PIPKI $\gamma$ and talin by proline-directed protein kinases. J Cell Biol. 2005;168(5):789-799.

18. Goult BT, et al. Structure of a double ubiquitin-like domain in the talin head: a role in integrin activation. EMBO J. 2010;29(6):1069-1080.

19. Gingras AR, et al. Structural determinants of integrin binding to the talin rod. J Biol Chem. 2009; 284(13):8866-8876

20. Gingras AR, et al. Central region of talin has a unique fold that binds vinculin and actin. J Biol Chem. 2010;285(38):29577-29587.

21. Gingras AR, et al. Structural and dynamic characterization of a vinculin binding site in the talin rod. Biochemistry. 2006;45(6):1805-1817.

22. Zhang X, Jiang G, Cai Y, Monkley SJ, Critchley DR Sheetz MP. Talin depletion reveals independence of initial cell spreading from integrin activation and traction. Nat Cell Biol. 2008;10(9):1062-1068.

23. Franco SJ, et al. Calpain-mediated proteolysis of talin regulates adhesion dynamics. Nat Cell Biol. 2004;6(10):977-983.

24. Nicolaou N, et al. Gain of glycosylation in integrin $\alpha 3$ causes lung disease and nephrotic syndrome. J Clin Invest. 2012;122(12):4375-4387.

25 . Has $C$, et al. Integrin $\alpha 3$ mutations with kidney, lung, and skin disease. $N$ Engl J Med. 2012; 366(16):1508-1514

26. Hasselbacher K, et al. Recessive missense mutations in LAMB2 expand the clinical spectrum of LAMB2-associated disorders. Kidney Int. 2006;70(6):1008-1012.

27. Chen YM, Kikkawa Y, Miner JH. A missense LAMB2 mutation causes congenital nephrotic syndrome by impairing laminin secretion. J Am Soc Nephrol. 2011;22(5):849-858.

28. Yao J, et al. $\alpha$-Actinin-4-mediated FSGS: an inherited kidney disease caused by an aggregated and rapidly degraded cytoskeletal protein. PLoS Biol. 2004;2(6):e167.

29. Brown EJ, et al. Mutations in the formin gene INF2 cause focal segmental glomerulosclerosis. Nat Genet. 2010;42(1):72-76.

30. Mele C, et al. MYO1E mutations and childhood familial focal segmental glomerulosclerosis. NEngl
JMed. 2011;365(4):295-306

31. Monkley SJ, et al. Disruption of the talin gene arrests mouse development at the gastrulation stage. Dev Dyn. 2000;219(4):560-574

32. Moeller MJ, Sanden SK, Soofi A, Wiggins RC, Holzman LB. Podocyte-specific expression of cre recombinase in transgenic mice. Genesis. 2003;35(1):39-42.

33. Debrand E, et al. Mice carrying a complete deletion of the talin2 coding sequence are viable and fertile. Biochem Biophys Res Commun . 2012;426(2):190-195.

34. Calderwood DA, Zent R, Grant R, Rees DJ, Hynes $\mathrm{RO}$, Ginsberg $\mathrm{MH}$. The Talin head domain binds to integrin $\beta$ subunit cytoplasmic tails and regulates integrin activation. J Biol Chem. 1999; 274(40):28071-28074

35. Czuchra A, Meyer H, Legate KR, Brakebusch C, Fassler R. Genetic analysis of $\beta 1$ integrin "activation motifs" in mice. J Cell Biol. 2006;174(6):889-899.

36. Wang P, Ballestrem C, Streuli CH. The $\mathrm{C}$ terminus of talin links integrins to cell cycle progression. J Cell Biol. 2011;195(3):499-513.

37. Morgan JR, et al. A role for talin in presynaptic function. J Cell Biol. 2004;167(1):43-50.

38. Soda K, et al. Role of dynamin, synaptojanin, and endophilin in podocyte foot processes. J Clin Invest. 2012;122(12):4401-4411.

39. Burkel BM, von Dassow G, Bement WM. Versatile fluorescent probes for actin filaments based on the actin-binding domain of utrophin. Cell Motil Cytoskeleton. 2007;64(11):822-832.

40. George B, et al. Crk1/2-dependent signaling is necessary for podocyte foot process spreading in mouse models of glomerular disease. J Clin Invest. 2012; 122(2):674-692.

41. Mundel P, Reiser J. Proteinuria: an enzymatic disease of the podocyte? Kidney Int. 2010;77(7):571-580.

42. Bate N, et al. Talin contains a C-terminal calpain2 cleavage site important in focal adhesion dynamics. PLoS One. 2012;7(4):e34461.

43. Peltier J, et al. Calpain activation and secretion promote glomerular injury in experimental glomerulonephritis: evidence from calpastatin-transgenic mice. J Am Soc Nephrol. 2006;17(12):3415-3423.

44. Chan KT, Bennin DA, Huttenlocher A. Regulation of adhesion dynamics by calpain-mediated proteolysis of focal adhesion kinase (FAK). J Biol Chem. 2010;285(15):11418-11426.

45. Serrano K, Devine DV. Vinculin is proteolyzed by calpain during platelet aggregation: $95 \mathrm{kDa}$ cleavage fragment associates with the platelet cytoskel- 
eton. Cell Motil Cytoskeleton. 2004;58(4):242-252.

46. Kelly BL, Ferreira A. $\beta$-Amyloid-induced dynamin 1 degradation is mediated by $\mathrm{N}$-methyl-D-aspartate receptors in hippocampal neurons. $J$ Biol Chem. 2006;281(38):28079-28089.

47. Kwak HI, et al. Calpain-mediated vimentin cleavage occurs upstream of MT1-MMP membrane translocation to facilitate endothelial sprout initiation. Angiogenesis. 2012;15(2):287-303.

48. Cortesio CL, Boateng LR, Piazza TM, Bennin DA, Huttenlocher A. Calpain-mediated proteolysis of paxillin negatively regulates focal adhesion dynamics and cell migration. J Biol Chem. 2011;286(12):9998-10006.

49. Lade A, Ranganathan S, Luo J, Monga SP. Calpain induces $\mathrm{N}$-terminal truncation of $\beta$-catenin in normal murine liver development: diagnostic implications in hepatoblastomas. J Biol Chem. 2012; 287(27):22789-22798.

50. Calderwood DA, Campbell ID, Critchley DR. Talins and kindlins: partners in integrin-mediated adhesion. Nat Rev Mol Cell Biol. 2013;14(8):503-517.

51 . Sachs N, et al. Blood pressure influences end-stage renal disease of Cd151 knockout mice. J Clin Invest. 2012;122(1):348-358

52. $\mathrm{Ma} \mathrm{H}$, et al. Inhibition of podocyte FAK protects against proteinuria and foot process effacement. JAm Soc Nephrol. 2010;21(7):1145-1156.

53. Chiluiza D, Krishna S, Schumacher VA, Schlöndorff J. Gain-of-function mutations in transient receptor potential C6 (TRPC6) activate extracellular-signal-regulated kinases Erk1/2. J Biol Chem.
2013;288(25):18407-18420.

54. Yu CC, et al. Abatacept in B7-1-positive proteinuric kidney disease. NEnglJ Med.2013;369(25):2416-2423.

55. Qu H, et al. Kindlin-2 regulates podocyte adhesion and fibronectin matrix deposition through interactions with phosphoinositides and integrins. J Cell Sci. 2011;124(pt 6):879-891.

56. Praekelt U, et al. New isoform-specific monoclonal antibodies reveal different sub-cellular localisations for talin1 and talin2. Eur J Cell Biol. 2012; 91(3):180-191.

57. Durocher Y, Perret S, Kamen A. High-level and high-throughput recombinant protein production by transient transfection of suspension-growing human 293-EBNA1 cells. Nucleic Acids Res. 2002; 30(2):E9.

58. Harita Y, et al. Phosphorylation of nephrin triggers $\mathrm{Ca}^{2+}$ signaling by recruitment and activation of phospholipase C $\gamma 1$. J Biol Chem. 2009;284(13):8951-8962.

59. Cong R, Li Y, Biemesderfer D. A disintegrin and metalloprotease 10 activity sheds the ectodomain of the amyloid precursor-like protein 2 and regulates protein expression in proximal tubule cells. Am J Physiol Cell Physiol. 2011;300(6):C1366-C1374.

60. Bouaouina M, Lad Y, Calderwood DA. The N-terminal domains of talin cooperate with the phosphotyrosine binding-like domain to activate $\beta 1$ and $\beta 3$ integrins. J Biol Chem. 2008;283(10):6118-6125.

61. Simonson WT, Franco SJ, Huttenlocher A. Talin1 regulates TCR-mediated LFA-1 function. J Immunol. 2006;177(11):7707-7714.
62. Petrich BG, et al. Talin is required for integrin-mediated platelet function in hemostasis and thrombosis. J Exp Med. 2007;204(13):3103-3111.

63. Nieswandt B, et al. Loss of talin 1 in platelets abrogates integrin activation, platelet aggregation, and thrombus formation in vitro and in vivo.J Exp Med. 2007;204(13):3113-3118.

64. Chen J, Chen MX, Fogo AB, Harris RC, Chen JK. $\mathrm{mVps} 34$ deletion in podocytes causes glomerulosclerosis by disrupting intracellular vesicle trafficking. J Am Soc Nephrol. 2013;24(2):198-207.

65. Ma W, et al. Calpain mediates pulmonary vascular remodeling in rodent models of pulmonary hypertension, and its inhibition attenuates pathologic features of disease. J Clin Invest. 2011;121(11):4548-4566.

66. Uceyler N, Biko L, Sommer C. MDL-28170 has no analgesic effect on CCI induced neuropathic pain in mice. Molecules. 2010;15(5):3038-3047.

67. Verderame M, Alcorta D, Egnor M, Smith K, Pollack R. Cytoskeletal F-actin patterns quantitated with fluorescein isothiocyanate-phalloidin in normal and transformed cells. Proc Natl Acad Sci U S A. 1980;77(11):6624-6628.

68. Boguslavsky S, et al. p120 catenin regulates lamellipodial dynamics and cell adhesion in cooperation with cortactin. Proc Natl Acad Sci U S A. 2007; 104(26): 10882-10887.

69. Perera RM, Zoncu R, Lucast L, De Camilli P, Toomre D. Two synaptojanin 1 isoforms are recruited to clathrin-coated pits at different stages. Proc Natl Acad SciU S A. 2006;103(51):19332-19337. 